\title{
FORMA DELLA NORMA-STATO E FATTO DEL POTERE PASTORALE ${ }^{1}$
}

\section{FORM OF THE STATE-NORM AND FACT OF THE PASTORAL POWER}

\author{
Alessia Magliacane \\ Université Paris 1 Panthéon-Sorbonne - (Paris, França)
}

Recebimento: 15 maio 2017

Aceitação: 10 ago. 2017

\begin{abstract}
Como citar este artigo / How to cite this article (informe a data atual de acesso / inform the current date of access):
MAGLIACANE, Alessia. Forma della norma-stato e fatto del potere pastorale. Revista da Faculdade de Direito UFPR, Curitiba, PR, Brasil, v. 62, n. 2, p. 175 - 208, maio/ago. 2017. ISSN 2236-7284. Disponível em: <http://revistas.ufpr.br/direito/article/view/52428>. Acesso $\quad$ em: 28 ago. $2017 . \quad$ DOI: http://dx.doi.org/10.5380/rfdufpr.v62i2.52428.
\end{abstract}

\section{RIASSUNTO}

La complessità del soggetto (individuale e collettivo) è sottoposta nella fase detta postmoderna (Harvey, Jameson, Raymond) ad un aggressivo tentativo di riduzione che ha come strumento la norma-stato, che ha attraversato pressoché indenne le epoche storiche fino alla modernità borghese capitalistica indagata da Habermas (nelle strutture discorsive), da Foucault (nella trasformazione del potere disciplinare e pastorale), da Deleuze (nella particolare struttura della ripetizione, erede della coazione freudiana), da Lacan (nella dialettica della repressione simbolico-normativa), da Butler (nella dialettica tra soggettivazione e assoggettamento) ma soprattutto nelle forme dell'immaginario letterario e cinematografico (di cui si fanno qui gli esempi di Lynch e McCarthy). L'autore tenta anche una netta distinzione tra la critica del diritto e l'analisi critica della forma normativa-giuridica, nella comparazione con altre esistenti forme normative quali quella strettamente simbolica (ad esempio nella morale sociale e nella famiglia) e quella istituzionale (ad esempio nelle forme di alienazione e di istituzione totale) alla luce delle più moderne teorie linguistiche, sistemiche, psicologiche e della complessità.

\section{PAROLE CHIAVE}

Complessità. Norma-stato. Postmodernismo. Potere pastorale. Psicanalisi.

\begin{abstract}
The complex structure of subjectivity in the Postmodernism (Harvey, Jameson, Raymond) is the object of an aggressive reduction through the tool of the state-norm, which traversed the many historical phases of the bourgeois modernity of Capitalism. Its discursive structure was analyzed by Habermas; the pastoral power was focused on Foucault's theory of discipline; the peculiar structure of the repetition - repetitiveness - was inherited by Deleuze from the Freudian category of coerciveness; Lacan enounced the dialectics of normative-symbolic repression; and Judith Butler supposed a double bind subjection-subjectivation. This feature of the state-norm is also investigated by popular culture and imaginary, of which we mention here the examples of David Lynch and Cormac McCarthy. The author seeks a clear distinction between the criticism of Law and the critical

\footnotetext{
${ }^{1}$ A Equipe Editorial da Revista da Faculdade de Direito UFPR agradece à Professora Doutora Angela Couto Machado Fonseca por seu auxílio editorial indispensável à publicação deste artigo [N. do E.]
} 
analysis of normative juridical forms, throughout a comparison with other normative forms: moral and family standards (a domain that is strictly symbolic), as well as institutions of normative structure (the domain of modern alienation). Methodologically, modern linguistic, systemic, psychology and complexity theories are also taken into account.

\section{KEYWORDS}

Complexity. State-norm. Postmodernism. Pastoral power. Psychoanalysis.

Se, nella teoria giusnaturalistica dello Stato, le persone si spogliano di tutta la loro autorità a favore dello Stato, ciò accade in base al presupposto (esplicitamente enunciato da Spinoza nel Trattato teologico-politico) che il singolo come tale, e prima della conclusione di questo contratto razionale, eserciti anche de iure ogni potere che detiene de facto. Forse queste concezioni sono state ravvivate, in seguito, dalla biologia darwiniana, che considera, in modo affatto dogmatico, insieme alla selezione naturale, solo la violenza come mezzo originario e solo adeguato a tutti i fini vitali della natura.

Benjamin, Per la critica della violenza, in Angelus Novus [2006: 6]

Può un mito essere "non-costruttivo", può immaginarsi, nell'ordine di intuizioni del Sorel, che sia produttivo di effettualità uno strumento che lascia la volontà collettiva nella sua fase primitiva ed elementare del suo mero formarsi, per distinzione (per "scissione") sia pure con violenza, cioè distruggendo i rapporti morali e giuridici esistenti? Ma questa volontà collettiva, così formata elementarmente, non cesserà subito di esistere, sparpagliandosi in una infinità di volontà singole che per la fase positiva seguono direzioni diverse e contrastanti?

GRAMSCI, Noterelle sulla politica del Machiavelli, in Quaderni dal carcere [1976: Quaderno 13: 17, 2320]

\section{INTRODUZIONE}

La proposta di queste brevi note introduttive è quella di una lettura della teoria discorsiva del diritto attraverso un prisma, per struttura sfaccettato e poliedrico, che non ripara e non protegge dal rischio di contraddizioni e di indeterminatezza: l'idea storico-sociologica della istituzione immaginaria della società. Senza ripercorrere in una sintesi necessariamente insufficiente la pluralità degli approcci ${ }^{2}$, l'idea di questa lettura comparata - che sfugge ai canoni della comparazione - nasce dopo la verifica delle insufficienze di una fase (finanche epocale) nella quale la produzione di sapere

\footnotetext{
${ }^{2}$ Facciamo riferimento agli anni '60 e '70 ed agli investimenti tematici, politici, libìdici che li hanno attraversati. Verranno qui riprese, ad esempio, le analisi di Foucault, Deleuze, Guattari, Lacan, Althusser, Castoriadis, Lyotard, Baudrillard, Bourdieu, Ricœur e (in forma di film) di Debord, Godard, Tarkovskij e (in forma di romanzo o sceneggiatura) Dick e McCarthy... agli anni '80 e '90, di Malick, Eastwood, Scorsese, Conte, Gould, e di Balibar, Cavell, Dussel, Habermas, Jameson e del nuovo millennio dei fratelli Coen, di Dominik, ancora di McCarthy e della "matrice" dei fratelli Wachoski e di Žižek, e di Lynch.
} 
sia riflessa e rielaborata dalla prassi. Attraverso l'idea, democratica nel suo svolgersi, della trasformazione della realtà. È l'esistenza di uno sguardo politico nel senso ampio del termine, secondo la bellissima ispirazione del poeta ${ }^{3}$, a mancare; uno sguardo che preesiste e coesiste all'azione, che è azione. Azione o possibilità d'azione per ciò stesso partecipata e creativa.

Questa lettura si pone, quindi, completa ed incompleta, come fugace sguardo di un’epoca, il cui occhio-tentativo è il movimento del farsi ed auto-organizzarsi della società.

Come una canzone del Novecento, cantata in un $d o d o^{4}$.

\section{FAKTIZITÄT UND GELTUNG 1992}

E o mesmo Padre Eterno que nunca foi lá / Olhando aquele inferno, vai abençoar / O que não tem governo, nem nunca terá / O que não tem vergonha, nem nunca terá / O que não tem juízo. Chico Buarque de Hollanda, O que será? (À flor da terra)

Il y a an et demi avait lieu un congrès à Bonneval qui est une petite ville, je crois, de la Sarthe, remarquable en ce sens qu'elle est dominée par une espèce d'immense bâtiment qui a toutes les apparences extérieures, de loin, d'un grand château, parce que c'est une plein rase; on voit ça de loin: c'est l'hôpital psychiatrique.

ALTHUSSER, Psychanalyse et sciences humaines [1996: 59]

La progressiva demitizzazione del linguaggio, che è solo un aspetto di tutto il processo illuministico, si rovescia in magia. HORKHEIMER - ADORNO, "L'industria culturale”, in Dialettica dell'illuminismo [1997: 178]

Au lieu de se désintégrer dans le pilier de la régulation, le pilier de l'émancipation a continué à briller, mais avec un éclat qui ne provenait plus de la tension dialectique initiale entre régulation et émancipation, mais des différents miroirs dans lesquels la régulation se reflète. Dans ce processus, l’émancipation a cessé d'être l'alter ego de la régulation pour devenir son double.

SOUSA SANTOS, Vers un nouveau sens commun juridique [2004: 9]

La ragione comunicativa subentra nelle procedure di razionalizzazione dell’azione giuridica.

L’effetto è esplosivo. Il collante dell'intesa linguistica e delle sue presupposizioni idealizzanti trova nella sfera giuridico-normativa della procedura argomentativa una capacità di espansione totalizzante. La sanzione, intesa come forza della legge ${ }^{5}$, rende fattuali quelle presupposizioni,

\footnotetext{
${ }^{3}$ Si muero,/ dejad el balcón abierto./ El nĩno come naranjas./(Desde mi balcón lo veo)./ El segador siega el trigo./(Desde mi balcón lo siento)./¡ Si muero, / dejad el balcón abierto! (Federico Garcia Lorca).

${ }^{4} \mathrm{Si}$ allude al motivetto che chiude l'album 900 di Paolo Conte [1992].

${ }^{5}$ La sanzione riflette anche il modo di decisione proprio al diritto, e cioè che le aspettative sociali, per acquisire una validità giuridica, devono essere filtrate e legalizzate per mezzo di un processo di decisione. Si veda, sulla legittimazione,
} 
controfattuali, che costituiscono l'intersoggettività della realtà del linguaggio. Ma nel fare ciò, non ne svela forse la contraddizione performativa? Quella di ogni formazione simbolica che cerchi di salvaguardare la tensione tra validità e fattualità?

Se la tensione esterna tra validità sociale e validità giuridica viene risolta attraverso la teoria dell'agire comunicativo, come può sussistere ancora una tensione interna al diritto?

È nell'atto dell'interpretare che il problema del simbolo si iscrive nella realtà.

Perché, allora, canalizzare nella procedura giuridica istituzionalizzata il senso performativo dell'agire comunicativo? E qual è il senso di questo surplus della realtà normativa della società?

L’argomentazione giuridica è la norma sociale dominante, premessa e risultante di questo incontro nel campo giuridico, idealizzato come libero autogoverno degli uomini. Il tribunale discorsivo, che viene in tal modo ad istituirsi, garantisce che ogni nuova pretesa di validità in grado di trasformarsi dal vero al reale possa trovare la propria forma giuridico-normativa, escludendo statutariamente dai processi di trasformazione il vero che non sia anche reale. Impedendo il sorgere dell' "anticipazione etica” che si ricostruisce a partire della solidarietà come l'altro lato del discorso pratico $^{6}$, e che nella filosofia della liberazione di Dussel si specifica nell'esteriorità della libertà dell’altro, una esteriorità escatologica, la "proximitad” con "sincronía atemporal”"

Il genere di norme che stiamo cercando dovrebbe pertanto produrre disponibilità all'obbedienza attraverso sia costrizione fattuale sia validità legittima. Norme di questo genere dovrebbero presentarsi con un'autorità capace di attribuire ancora una volta alla validità la forza del fattuale, questa volta però a partire dalla già avvenuta polarizzazione tra agire orientato al successo e agire orientato all'intesa, e dunque a partire da una già percepita inconciliabilità di fattualità e validità ${ }^{8}$.

LuHManN, Procedimenti giuridici e legittimazione sociale [1995], ma anche I diritti fondamentali come istituzione [2002], e, sulla legittimità, DERRIDA, Force de loi [1994].

${ }^{6}$ HABERMAS, Teoria dell'agire comunicativo [1997].

${ }^{7}$ DusSEL, Filosofía de la Liberación [1977].

${ }^{8}$ HABERMAS, Fatti e Norme [1996: 37]. Che questa impostazione possa presupporre, nel Discorso di Intesa, un'ideologia del consenso, come suggerisce LYOTARD, La condition postmoderne [1979: 105, n. 227], è questione che affronteremo in seguito. "En toute société, montrera Spinoza, il s'agit d'obéir et rien d'autre: c'est pourquoi les notions de faute, de mérite et de démérite, de bien et de mal, sont exclusivement sociales, ayant trait à l’obéissance et à la désobéissance. La meilleure société sera donc celle qui exempte la puissance de penser du devoir d'obéir, et se garde en son propre intérêt de la soumettre à la règle d'Etat, qui ne vaut que pour les actions. Tant que la pensée est libre, donc vitale, rien n'est compromis; quand elle cesse de l'être, toutes les autres oppressions sont aussi possibles, et déjà réalisées, n'importe quelle action devient coupable, toute vie menacée”, Deleuze, Spinoza [1970: 6]. E il principio del parallelismo spinoziano, per cui “l'ordine e la connessione delle idee coincide con l'ordine e la connessione delle cose” (SPINOZA, Etica, Parte seconda, proposizione VII [2005: 134]), “n’implique aucune dévalorisation de la conscience à l'étendue, mais, ce qui est beaucoup plus important, une dévalorisation de la conscience par rapport à la pensée: une découverte de l'inconscient, et d'un inconscient de la pensée, non moins profonds que l'inconnu du corps” (DeLEuzE, 1970: 23). Così, sul piano giuridico, "the only way to save flexibility in accord with ontology and epistemology is to see law as some sort of deficient knowledge. [...] And if the stimulus of collective efforts is fear and hope, then the main tool for a conscious collective effort is law, that is, prescriptive discourse backed by sanctions and compensations. It becomes a necessary tool for political stability as long as we are not able to appreciate our utility both at individual and collective levels with sufficient scientific method and knowledge. Norms do not direct human conduct, they may be taken instrumentally to meliorate the 
In questa "già percepita inconciliabilità di fattualità e validità" si iscrive l'estensione (nascosta) della realtà simbolica della teoria discorsiva del diritto, e dunque la funzione (espressa) di mediazione del diritto, che annulla questa tensione, nell'espressione (sanzione) di non-immediatezza alla realtà. Ciò riporta alla riformulazione dell'esperienza del sacro e ad alla centralità dell’epistemologia del simbolo9. "È la stessa ambivalenza che domina in genere il rapporto con il padre”.

Dice Freud, (con)fondendo la forma storica del potere normativo con il modello ricorrente del nome del padre.

\begin{abstract}
"Sacer" significa non solo "sacro", "consacrato", ma anche qualcosa che possiamo tradurre soltanto con "infame", "esecrando" (auri sacra fames). Tuttavia la volontà del padre non era soltanto qualcosa di intoccabile, qualcosa da tenere altamente in onore, ma anche qualcosa di fronte a cui si tremava, perché esigeva una dolorosa rinuncia pulsionale ${ }^{10}$.
\end{abstract}

Con Foucault la legge codifica la norma sociale e risponde ad una razionalità propria, che è la raison d'État, nelle sue forme economico-giuridiche e manifestazioni storiche. Con Habermas, la norma giuridica - non più solo legge - trova nel consenso razionalmente fondato e argomentato della comunità interpretativa la base fattuale della sua pretesa di validità ideale.

Riprendendo la metafora fondatrice, l'usura dell'office du père si rivela a favore di un ordine in cui l'individuo fonda se stesso e si pone come istanza sovrana, legislatore e giudice di tabù ${ }^{11}$, ma anche testimone del processo di verità contenuto nella digressione di Dialettica dell'illuminismo “Juliette, o illuminismo e morale”. La ragione, infatti, “organo del calcolo e della pianificazione”, in virtù del suo carattere formale, “neutra rispetto ai fini e a disposizione di tutti gli interessi naturali” -

orientation of conduct by default”, PfERsMAnN, Law's normativity in Spinoza's naturalism [2004: 652 e 655]. “In conclusione: un patto non ha nessuna forza e nessun valore se non in ragione dell'utilità che procura ai contraenti: tolta questa, viene inficiato nello stesso tempo il patto che cade in stato di nullità. Perciò si comporterebbe in modo insensato chi richiedesse ad altra persona la sua parola in perpetuo, se al tempo stesso non si adoperasse a far sì che alla rottura del patto da concludersi possa seguire più danno che vantaggio a chi prende l'iniziativa della rottura”, SPINOZA, Trattato teologico-politico [2005: 648]. Il corsivo è nostro.

${ }^{9}$ RICEUR, De l'interprétation [1965: 13]. Sul piano sociologico, BouRDIEU, Langage et pouvoir symbolique [2001: 155].

${ }^{10}$ FreUd, L'uomo Mosé [2006: 439].

${ }^{11} \mathrm{Ne}$ analizza gli atti-effetti del processo LEGENDRE, Le crime du caporal Lortie [2000], ma già, storicamente, FOUCAULT, Io, Pierre Rivière [2000]. Sulla metafora fondatrice, ovviamente, soprattutto, LACAN, Introduction aux noms-du-Père [2005], ma già, didatticamente, FrEUD, L'avvenire di un'illusione [1975]. Sull'Edipo lacaniano e la crisi del simbolico, si veda BorCh-JACOBSEN, Lacan. Le maître absolu [1995]. "Partout, autrement dit, s'étale la crise de l'identification symbolique et l’impossibilité de la séparer, comme le voudrait Lacan, de l’identification dite 'imaginaire’. [...]. L’Edipe lacanien, ce n'est pas l'Edipe tel qui est, c'est l'Edipe tel qu'il doit être. C'est l'Edipe anti-mimique, l'anti-modèle identificatoire auquel l'analyste, par son silence, enjoint à l'analysant de se conformer: 'Identifie-toi à mon désir', '(Ne) soit (pas) comme moi', 'Imite l'inimitable'. C'est pourquoi d'ailleurs le 'modèle' de Lacan n'était pas l' Edipe roi, l’Edipe névrosé qui 'subi(t) l’interdit' tout en rivalisant avec lui, mais bien l’Edipe à Colone, l’Edipe qui 'encour(t) la castration, s’arrache volontairement les yeux et fulmine, irréconcilié, contre la malédiction d'exister'”. Così BoRCHJACOBSEN [1995: 268]. 
il suo unico elemento è la coordinazione -, non lascia operare nessuna altra determinazione che le classificazioni dell'attività sociale ${ }^{12}$.

Sul gioco di questa oscura battaglia Pierre Rivière scrive il suo racconto-delitto, "ed è per questa via che egli lo faceva comunicare con la storia dei delitti sacrificali e gloriosi, o piuttosto che compiva con le sue mani un delitto storico"13.

Quindici anni prima del testo lacaniano ${ }^{14}$, Adorno aveva già mostrato come i personaggi di Sade erano spinti dall'obbedienza incondizionata alla pura forma di una Legge morale strutturalmente identica all’imperativo categorico kantiano. “Juliette non incarna né una libido non sublimata, né una libido regressiva, ma il godimento intellettuale della regressione, l'amor intelectualis diaboli, il piacere di distruggere la civiltà con le proprie armi”"15.

Così,

via the reference to Sade, Lacan reads absence as an act of rendering invisible, of "repressing", the moral Law's enunciator, and it is Sade who renders it visible in the figure of the "sadist" executioner - torturer - this executioner is the enunciator of the moral Law, the agent who finds pleasure in our (the moral subject's) pain and humiliation ${ }^{16}$.

Scrive ancora Foucault, nel corso al Collège de France del 14 gennaio 1976:

Le discours de la discipline est étranger à celui de la loi; il est étranger à celui de la règle comme effet de la volonté souveraine. Les disciplines vont donc porter au discours qui sera celui de la règle, mais non pas de la règle juridique dérivée de la souveraineté; elles porteront un discours de règle naturelle, c'est-à-dire de la norme. Elles définiront un code qui sera celui, non pas de la loi, mais de la normalisation, et elles se référeront néces-sairement à un horizon théorique qui ne sera pas l'édifice du droit mais le champ des sciences humaines, et leur jurisprudence sera celle d'un savoir clinique ${ }^{17}$.

\footnotetext{
12 "La mitologia specifica di cui l’illuminismo occidentale (anche in forma di calvinismo) doveva fare tabula rasa, era la dottrina cattolica dell'ordo e la religione popolare pagana che continuava a fiorire alla sua ombra”, HORKHEIMER ADORNO, “Juliette, o illuminismo e morale”, in Dialettica dell’illuminismo [1997: 95-96].

${ }^{13}$ FOUCAULT, Io, Pierre Rivière [2000: 226].

${ }^{14}$ LACAN, Kant avec Sade [1962, 1999]. Sul testo lacaniano come punto di torsione del pensiero del suo autore, SAFATLE, L'acte au-delà de la Loi [2002: 94].

${ }^{15}$ HORKHEIMER - ADORNO, “Juliette, o illuminismo e morale”, in Dialettica dell’illuminismo [1997: 106]. "Même là, dans la perversion où le désir, en somme, apparaîtrait en se donnant pour ce qui fait la loi, c’est-à-dire pour une subversion de la loi, il est en fait bel et bien le support d’une loi”, LACAN, Séminaire X, séance non pubblicata del 27/02/1961.

16 ŽIŽEK, Kant and Sade: the ideal couple [2001], http://lacan.com/frameXIII2.htm. "So the main effort of Lacan's seminar on the Ethics of Psychoanalysis is precisely to break up the vicious cycle of Kant avec Sade. How is this possible? Only if - in contrast with Kant - one asserts that the faculty of desiring is not in itself 'pathological'. In short, Lacan asserts the necessity of a 'critique of pure desire': in contrast to Kant, for whom our capacity to desire is thoroughly 'pathological' (since, as he repeatedly stresses, there is no a priori link between an empirical object and the pleasure this object generates in the subject), Lacan claims that there is a 'pure faculty of desire', since desire does have a nonpathological, a priori object - cause - this object, of course, is what Lacan calls objet petit a”. Sull'etica kantiana, ŽIžEK, The indivisible remainder [1996, in particolare il capitolo secondo].

${ }^{17}$ FOUCAULT, Dits et écrits II [2001: 175]. Contro la lettura dell'incompatibilità tra potere giudiziario e potere disciplinare nell'ambito del paradigma della modernità, che nasconde le profonde interpenetrazioni tra i due poteri, e lo scivolamento naturale dalla scienza al diritto all'interno delle medesime istituzioni, SoUSA SANTOS, Vers un nouveau sens commun juridique [2004, e in particolare la prima parte: "Vers une transition paradigmatique dans la science et dans le droit”]. “A
} 


\section{Ci troviamo ad un nuovo tornante?}

Il passaggio dalle strutture dell'episteme registrata in prima linea dalle ricerche foucaultiane - dalla società disciplinare alla società del controllo - a quelle habermasiane di fine "secolo breve" dalla società del controllo a quella dell'autodisciplina, passaggio dall’istituzione al contratto? E cioè - anche sulla base delle convincenti ricerche di Habermas degli anni '70, con una radicalità che pure non toglie nulla all'efficacia giuridica delle ricostruzioni sulle trasformazioni del potere istituzionale - alla materializzazione istituzionale di nuove strutture di razionalità, che escludono, per ipotesi, dai processi di trasformazione le nuove possibilità strutturali in cui la ragione si scopra presso di sé (nella non-ragione) come non-ragione, e che regolano il passaggio dall'alienazione come processo all'atto di alienazione di sé ${ }^{18}$.

mon avis, ce qui caractérise le plus profondément la condition socioculturelle de cette fin de siècle c'est l'absorption du pilier de l'émancipation dans celui de la régulation, phénomène résultant de la gestion reconstructive des excès et des déficits de la modernité confiée à la science moderne, et, faute de mieux, au droit moderne. La colonisation progressive des différentes rationalités de l'émancipation moderne par la rationalité cognitive-instrumentale de la science, conduisit à la concentration des énergies émancipatrices et des capacités de la modernité dans la science e la technologie” (SouSA SANTOS, 2004: 27).

18 "Les sociétés peuvent faire un apprentissage évolutionnaire en utilisant pour une réorganisation de leurs systèmes d'action les potentiels cognitifs contenus au sein des visions du monde. On peut se représenter ce processus comme la matérialisation institutionnelle de structures de rationalité qui avaient déjà pris forme au sein des visions du monde”, HABERMAS, "Pour une reconstruction du matérialisme historique”, in Après Marx [1997: 85]. In termini filosofici si rimane ancorati all'idealismo hegeliano della storia. La disciplina, da contro-diritto, come contropartita politica delle norme giuridiche per disequilibrare le relazioni di potere, diviene il contenuto effettivo e istituzionalizzato delle forme giuridiche (FOUCAULT, Sorvegliare e punire, 1976). Ma, “cette réduction elle-même [...], la réduction de tous les éléments qui font la vie concrète d'un monde historique [...] à un principe d'unité interne, cette réduction n'est elle-même possible qu'à la condition absolue de tenir toute la vie concrète d'un peuple pour l'extériorisation aliénation (Entäusserung Entfremdung) d'un principe spirituel interne, qui n'est jamais rien d'autre en définitive que la forme la plus abstraite de la conscience de soi de ce monde: sa conscience religieuse ou philosophique, c'est-à-dire sa propre idéologie”, AlthUSSER, “Contradiction et surdétermination”, in Pour Marx [1965, 2005: 102]. 

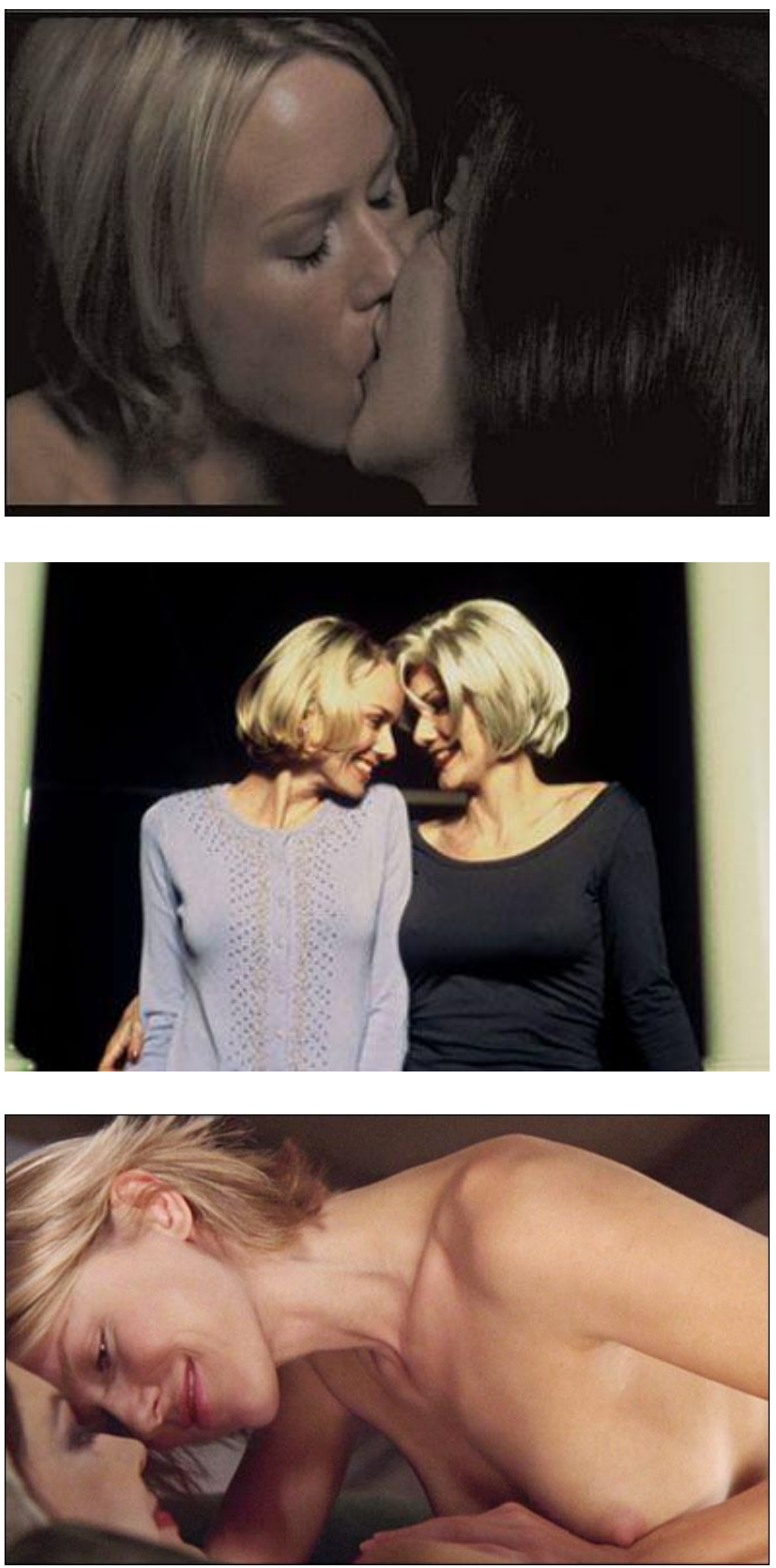

LYNCH, Mulholland Drive (Usa/Fra, 2001)

\section{SACHER-MASOCH 1967}

La propre nuit de son départ (car elle partit avant le jour), il me sembla, durant mon sommeil, que je la voyais garder nos moutons, et qu'un grand loup étant venu pour emporter la plus belle brebis du troupeau, ma pauvre sœur l'avait voulu empêcher, et qu'il l'avait emportée elle-même; et comme je courais après pour la délivrer, le loup fut changé en homme, et je vis Ursule le caresser. Et j’avais beau lui crier: «Ursule ! Ursule! C’est un loup! », elle ne m'écoutait pas, jusqu'au moment où étant redevenu loup, il l'avait dévorée. Je n’ai pas 
foi aux rêves; mais je rapporte celui-là à cause de sa singularité à pareil jour.

RETIF DE LA BRETONNE, La paysanne pervertie [1784, 1972: 4849]

Se il fantasma sadico riposa su un elemento ultimo, che Klossowski ha profondamente analizzato, il tema del padre distruttore della propria famiglia, che spinge la figlia a assassinare la madre, pater sive Natura prima ${ }^{19}$, è l'immagine della madre, invece, ad emergere nella sua qualità di complice del discorso masochista.

Così, dice Deleuze, quando la psicanalisi, anche nelle sue esplorazioni più avanzate, lega l'instaurazione di un ordine simbolico al nom du père, non è forse mantenere l'idea, poco analitica e, forse, prefreudiana, che la madre è dell'ordine della natura, e il padre, invece, solo "principio di cultura” e rappresentante della legge?

Il masochista vive l'ordine simbolico come inter-maternale, e pone dunque le condizioni che permettono alla madre, in questo ordine, di confondersi con la legge. Nel discorso masochista non c’è nessuna identificazione alla madre, poiché la madre non è il termine dell’identificazione, ma la condizione del simbolismo attraverso cui il masochista si esprime. In questo ordine il padre è già soppresso, annullato di ogni traccia che sia registrabile nel tempo.

Tuttavia il padre annullato nell'ordine simbolico continua ad agire nell'ordine del reale, secondo la nota legge enunciata da Lacan: ciò che viene abolito simbolicamente risorge nel reale nella sua forma allucinatoria ${ }^{20}$. La fine de La Vénus à la fourrure, di Sacher-Masoch, infatti, marca questo ritorno aggressivo e allucinante del padre, in un mondo che l'aveva annullato simbolicamente, ritorno che rende impossibile la continuazione del fantasma. Ma, sottolinea Deleuze, sarebbe un errore confondere il fantasma, che gioca nell’ordine simbolico, con l'allucinazione che manifesta invece la rivincita del vissuto nell’ordine del reale.

Loin d'être la vérité du masochisme, loin de sceller son alliance avec le sadisme, le retour offensif de l'image de père marque le danger toujours présent qui menace de l'extérieur le monde masochiste, et qui fait craquer les «défenses» que le masochiste a construites comme conditions et limites de son monde pervers symbolique ${ }^{21}$.

\footnotetext{
${ }^{19}$ KLOSSOWSKI, Eléments d'une étude psychanalytique sur le marquis de Sade [1933].

${ }^{20}$ Lacan definisce la forclusion (Verwerfung) come un meccanismo che si esercita nell'ordine simbolico e che porta essenzialmente sul padre, o, piuttosto, sul nom du père. "Il semble que Lacan considère ce mécanisme comme original, indépendant de toute étiologie maternelle (la défiguration du rôle de la mère serait plutôt l'effet de l'annulation du père dans la forclusion). Toutefois, dans la perspective de Lacan, l'article de Pierre Aulagnier, Remarques sur la structure psychotique, La Psychanalyse, VIII, qui semble restituer à la mère un certain rôle d'agent symbolique actif”, DELEUZE, Présentation de Sacher-Masoch [1967: 57, nota 17].

${ }^{21}$ DeLeuze, Présentation de Sacher-Masoch [1967: 58]. "Theodor Reik cite un cas où toute la «magie» de la scène masochiste s'évanouit, parce que le sujet a cru voir dans la femme prête à le frapper quelque chose qui lui rappelait le père. [...]. Reik commente ce cas comme s’il prouvait que le père est bien la vérité de la femme bourreau, qu'il est déguisé dans l'image de mère; Reik en tire un argument pour l'unité sado-masochiste. Nous croyons qu'il faut en tirer des
} 
Che fare allora per premunirsi contro tale ritorno? L'eroe di Masoch deve utilizzare necessariamente una procedura complessa per proteggere il suo mondo fantasmatico e simbolico dagli attacchi allucinatori del reale (o dagli attacchi reali dell’allucinazione).

Questa procedura assume la forma del contratto.

Par le contrat, c'est-à-dire par l'acte le plus rationnel et le plus déterminé dans le temps, le masochiste rejoint les régions les plus mythiques et les plus éternelles - celles où règnent les trois images de mère. Par le contrat, le masochiste se fait battre; mais ce qu'il fait battre en lui, humilier et ridiculiser, c'est l'image du père, la ressemblance du père, la possibilité d'un retour offensif du père. Ce n'est pas «un enfant», c'est un père qui est battu. Le masochiste se rend libre pour une nouvelle naissance où le père n’a aucun rôle ${ }^{22}$.

Il contratto masochista ha dunque per significato quello di conferire il potere simbolico della legge all'immagine della madre, operando così una doppia dénégation: quella positiva, ideale e magnificante della madre (identificata alla legge) e quella negativa, annullante del padre (espulso dall'ordine simbolico), a differenza del sadismo, che presenta invece una negazione attiva della madre e un'inflazione del padre, al di sopra delle leggi. Questo processo formale in atto nell'universo masochista - il rinvio della legge alla madre, l'identificazione della legge all'immagine ideale della madre (la madre orale del Sacher-Masoch di Deleuze) - è la condizione che permette alla punizione di compiere la sua funzione originale, e alla colpevolezza di trasformarsi in trionfo.

Attraverso il contratto, infatti, il masochista raggiunge l'elemento impersonale di un destino che si esprime attraverso il mito, instaurando per un tempo determinato ciò che è contenuto, ritualmente, in ogni tempo nell'ordine simbolico del masochismo. "Du contrat au mythe, par l’intermédiaire de la loi: celle-ci sort du contrat, mais nous jette dans les rites”23.

Attraverso il contratto che esclude il padre, l'applicazione della legge paternale è rimessa nelle mani della madre, ed è tutta la legge a cambiare di segno, e a ordinare ciò che, dal punto di vista

conséquences contraires. Le sujet, dit Reik, est «désillusionné»; il faudrait dire qu’il est «dé-phantasmatisé», mais en revanche halluciné, hallucinisé” (DELEUZE, 1967: 58).

22 Deleuze, Présentation de Sacher-Masoch [1967: 58-59]. Il transfert delle funzioni paternali sulle tre immagini di madre (uterina, orale, edipica) non è che un primo aspetto, ci dice Deleuze, del fantasma masochista, che trova significato e completamento in un altro elemento: la condensazione di tutte le funzioni maternali sulla seconda madre, la madre orale, la "bonne mère”. Così, “c'est un erreur de mettre le masochisme en rapport avec le thème de la mauvaise mère. De mauvaises mères, il y en a dans le masochisme: la mère utérine, la mère œdipienne, les deux extrêmes du pendule. Mais tout le mouvement du masochisme est d'idéaliser les fonctions des mères mauvaises en les reportant sur la bonne mère” (DELEUZE, 1967: 55).

${ }^{23}$ DELEUZE, Présentation de Sacher-Masoch [1967: 89]. 
del padre, in quanto legata (la legge) all’immagine di padre, (la legge) è supposta difendere. La legge, adesso, è dunque completamente maternale ${ }^{24}$.

Deleuze traccia così la cartografia del rapporto tra norma (in ultima istanza norma-stato) e fatto (in ultima istanza simbolico-normativo del potere), nella presentazione di Sade e di SacherMasoch come critici moderni dell’immagine moderna della legge, cioè l’immagine kantiana.

Infatti,

en même temps que la loi ne peut plus se fonder sur le Bien comme sur un principe supérieur [immagine platonica classica della legge], elle ne doit plus davantage se faire sanctionner par le Mieux comme bonne volonté du juste [critica classica socratica].

LA LOI, définie par sa pure forme, sans matière et sans objet, sans spécification, est telle qu'on ne sait pas ce qu'elle est, et qu'on ne peut pas le savoir. Elle agit sans être connue. Elle définit un domaine d'errance où l'on est déjà coupable, c'est-à-dire où l'on a déjà transgressé les limites avant de savoir ce qu'elle est: ainsi Edipe ${ }^{25}$.

Il fondamento impossibile della legge kantiana si trova così nel principio del Male dell’eroe sadico, male naturale, non morale, dove la legge ne costituisce l'espressione più completa. Sade infatti, secondo Deleuze, è il pensatore della riconciliazione della legge con la natura: è la perversione della natura seconda, artificiale e tirannica, espressa dalla legge, che permette in un crudele paradosso di ritrovare la natura primaria. Per Sade si tratta allora di reinventare una fondazione che serva a défoncer la legge, portando l’idea della legge stessa ad un punto di ironia, che potrebbe anche essere il suo punto più alto di verità, quello delle istituzioni perfette perché prive di leggi che leghino le azioni, che le immobilizzino e le moralizzino. Pure istituzioni senza legge sarebbero per natura dei modelli d'azioni libere, anarchiche, in perpetuo movimento, in rivoluzione permanente ${ }^{26}$.

Partendo quindi dall’idea di una legge che non possa essere fondata dal Bene, ma che deve riposare solo sulla sua pura forma, l'eroe sadico inventa una nuova maniera di rimontare dalla legge a un principio superiore; ma questo principio è l'elemento informale di una Natura primaria distruttrice delle leggi stesse.

Il y a chez Sade une profonde pensée politique, celle de l'institution révolutionnaire et républicaine, dans sa double opposition à la loi et au contrat. Mais cette pensée de l'institution, d'un bout à l'autre, est ironique, parce que sexuelle et sexualisée, montée en provocation contre toute tentative contractuelle et légaliste de penser la politique.

\footnotetext{
24 "Mais du point de vue de l'image de mère, au contraire, la castration du fils est la condition du succès de l'inceste, maintenant assimilé par ce déplacement à une seconde naissance où le père n'a pas de rôle ", DELEUZE, Présentation de Sacher-Masoch [1967: 81].

${ }^{25}$ DeLeuze, Présentation de Sacher-Masoch [1967: 73].

${ }^{26}$ Ne La Philosophie dans le boudoir emerge tutta la critica di Sade verso l'ideologia rivoluzionaria nel suo complesso, cioè, verso una concezione contrattuale del regime repubblicano e, soprattutto, verso l'idea della legge. È nella Rivoluzione francese, infatti, che Sade ritrova ciò che più odia: la legge e il contratto, che separano ancora i francesi dalla vera repubblica (Français, encore un effort si vous voulez être républicains).
} 
Ne faut-il pas attendre de Masoch un prodige inverse ? Non plus une pensée ironique, en fonction de la Révolution de 1789, mais une pensée humoristique en rapport avec les révolutions de 1848 ? Non plus une pensée ironique de l'institution dans son opposition avec le contrat et la loi, mais une pensée humoristique du contrat et de la loi, dans leurs rapports mutuels ? ${ }^{27}$

L’humour masochista di Sacher-Masoch - qualcosa di più complesso della metafora invertita di Rétif de la Bretonne dell'agnello che accarezza il lupo (che di lì a poco potrebbe divorarlo), ma anche qualcosa di meno eversivo ${ }^{28}$ - non cerca di fare della legge qualcosa di diverso rispetto a quello che già è, poiché non può aversi piacere masochista che in un contesto kantiano. Un contesto, cioè, formale, in cui la punizione preesista ad ogni colpa e dove l'applicazione della punizione stessa ne costituisca precisamente la sovversione. Poco importa quale sia la colpa commessa, e poco importa anche che una colpa sia stata commessa, pourvu que la loi prétende le contraire. "On prend la loi au mot, à la lettre; on ne conteste pas son caractère ultime ou premier; on fait comme si, en vertu de son caractère, la loi se réservait pour soi le plaisir qu'elle nous interdit”29.

Partendo quindi dall'altra scoperta moderna, la legge come processo punitivo, l'eroe masochista inventa una nuova maniera questa volta di "scendere" dalla legge alle sue conseguenze: rovesciare la colpevolezza e fare della punizione la condizione che renda possibile il piacere difeso. La sofferenza infatti non è la causa del piacere, ma è la condizione preliminare indispensabile alla venuta del piacere ${ }^{30}$.

È il contenuto edipico che subisce, in ultima istanza, una doppia trasformazione. La complementarietà madre-padre viene infatti infranta due volte, senza simmetria: dalla facoltà speculativa-dimostrativa (e dunque dalla reteirazione quantitativa) del discorso sadico, e dalla facoltà dialettica-immaginativa (e dunque dalla sospensione qualitativa) del discorso masochista ${ }^{31}$.

Come in A piece of monologue in Beckett:

\footnotetext{
${ }^{27}$ DeLeuZE, Présentation de Sacher-Masoch [1967: 70-71].

${ }^{28}$ Ci riferiamo a RETIF DE LA BRETONNE, La paysanne pervertie [1784, 1972: 49].

${ }^{29}$ DeLEUZE, Présentation de Sacher-Masoch [1967: 77]. L'humour masochista, scrive Deleuze, è di questo tipo: “la même loi qui m’interdit de réaliser un désir sous peine d'une punition conséquente est maintenant une loi qui met la punition d'abord et m’ordonne en conséquence de satisfaire le désir” (DELEUZE, 1967: 78).

${ }^{30}$ REIK, Le Masochisme [1953].

31 Deleuze, Présentation de Sacher-Masoch [1967: 79]. Riportiamo per completezza l'opinione, che tuttavia non condividiamo, di Laurent DE SUTTER, Deleuze. La pratique du droit [2009], e cioè che attraverso l'humour masochista operi già la critica della psicoanalisi lacaniana elaborata nell’Anti-Edipo dalla coppia Deleuze-Guattari, poiché la caduta della legge morale forse non è altro che la caduta della Legge simbolica che Lacan poteva ancora trovare in Sade: "Etablir l'équation Sade=Kant, c'est encore faire confiance à l'hypothèse d'une Loi qui sous-tendrait la nullité de tous les lois: une forme de la forme de loi, elle-même vide, comme l'est le Grand Autre lacanien. En revanche établir une équivalence entre Sacher-Masoch et Kant est bien plus explosif: cela revient à dire que, dans le ciel kantien aussi bien que dans l'enfer sadien, la forme de la loi est sans espoir, et que cette absence d'espoir s'applique à tout ce qui pourrait la racheter” (DE SUTTER, 2009: 32).
} 
There was father. That grey voice.

There mother. That other ${ }^{32}$.

\section{STRUTTURA DEL SENTIMENTO NEL DISCORSO POSTMODERNISTA 1991-2007}

Il diritto postmoderno, fondato sull'Etica del discorso, a cui possiamo estendere la stessa “struttura dei sentimenti” che Jameson attribuiva alla cultura, è un nuovo diritto interamente statuito, che prevede un sistema di regole per cui il rischio di dissenso, intrinseco all'agire comunicativo, sia circoscritto, in quanto destabilizzante l'integrazione sociale, ma abbia possibilità di esprimersi, in quanto forza auto-legittimante il diritto stesso ${ }^{33}$.

Se il postmodernismo, in altri termini,

corrisponde a quanto intendeva Raymond Williams con la sua fondamentale categoria culturale della "struttura del sentimento" (la quale, per adoperare un'altra delle categorie essenziali di Williams, è divenuta a sua volta "egemonica”), allora non può godere di tale status se non a forza di una profonda autotrasformazione collettiva, della rielaborazione e della riscrittura di un sistema precedente ${ }^{34}$.

In altri termini,

on comprend bien quelle est la fonctionne de ce recours dans l'argumentation de Habermas contre Luhmann. Le Diskurs y est l'ultime obstacle opposé à la théorie du système stable. La cause est bonne, mais les arguments ne les sont pas. Le consensus est devenu une valeur désuète, et suspecte. Ce qui ne l'est pas, c'est la justice. Il faut donc parvenir à une idée et à une pratique de la justice qui ne soit pas liée à celles du consensus ${ }^{35}$.

Una critica di carattere formale, diffusa soprattutto tra i filosofi del diritto, è ben espressa da Rosenfeld in questi termini:

Basandosi sul concetto di agire comunicativo, l'approccio proceduralista di Habermas affronta il residuo di arbitrarietà della democrazia facendo appello al consenso dialogico come fonte della legittimità del diritto. D’altro canto, il proceduralismo di Habermas garantisce ai diritti fondamentali un fondamento giuridico, che sembra eliminare ogni

\footnotetext{
32 Su cui DiAMOND, Feminist readings of Beckett [2004: 45].

33 Dunque, secondo le regole di riconoscimento dell'Altro nel sistema, solo in quanto Differenza-nell’Identità. "La vittima, l' 'Altro' appare come altro rispetto alla 'normalità': il sistema normale, 'naturale', 'buono', appare ora come il ‘capitale feticizzato' di Marx, come la 'Totalità' eticamente perversa di Levinas, la 'non-verità' di Adorno, e pertanto formalmente e intersoggettivamente perderà la sua validità, la propria egemonia (direbbe Gramsci)”. Così, "agli occhi delle vittime, dei dominati e/o esclusi si manifesterà solo come repressivo, come la 'anti-validità dominatrice"”, DussEL, Sei principi dell'architettonica dell'etica della liberazione [1999: 233]. "Si comprende come il potere della norma funzioni facilmente all'interno di un sistema di uguaglianza formale, poiché all’interno di una omogeneità che è la regola, esso introduce, come imperativo utile e risultato di una misurazione, tutto lo spettro delle differenze individuali”, FOUCAULT, Sorvegliare e punire [1976: 202].

34 JAMESON, Postmodernismo [2007: 10]. "Né si deve pensare che la questione della ricatalogazione e della transcodifica non abbia una sua particolare rilevanza: la funzione attiva - l'etica e la politica - di siffatti neologismi sta nella nuova impresa che propongono, cioè nella riscrittura di tutte le cose familiari in termini nuovi e quindi nella proposta di modificazioni, di nuove prospettive ideali, del rimescolamento di sentimenti e valori canonici” [2007: 10].

${ }^{35}$ LYOTARD, La condition postmoderne [1979: 106].
} 
necessità di giustificare tali diritti in base ad una particolare concezione del bene non condivisa da tutti i membri della comunità politica. Inoltre, l'approccio procedurale di Habermas al diritto non si limita ad offrire un modo per risolvere il conflitto tra democrazia e giustizia, ma aspira altresì a stabilire una connessione interna tra sovranità popolare e diritti umani, elaborando così una base normativa per un regime giuridico che intenda soddisfare sia la democrazia sia la giustizia. In ultima analisi, la possibilità di realizzare giustizia procedurale pura dipende dalle assunzioni di sfondo e dalle condizioni materiali che circondano l'elaborazione e l'uso delle pratiche e degli strumenti procedurali rilevanti. Di conseguenza, il paradigma procedurale del diritto di Habermas non riesce in fin dei conti a generare giustizia procedurale pura, e non è in grado di fornire una soluzione comprensiva per il conflitto tra democrazia e giustizia ${ }^{36}$.

La critica di Apel è rivolta invece alla nuova architettonica della "biforcazione” quale risulta in Faktizität und Geltung, in cui è posto un principio di discussione "moralmente neutro", e non più, come ancora nelle Tanner Lectures, il principio dell' “etica della discussione” come principio di fondazione di tutte le norme. Critica che verte sulla adeguatezza del principio fondamentale di discussione "moralmente neutro" a fondare la diversità dei due tipi di norme che per i due filosofi si tratta pur sempre di distinguere in una filosofia della discussione (norme della morale e norme del diritto) ${ }^{37}$. "Désormais, le «principe moral» et «le principe juridique» doivent procéder de manière cooriginaire du «principe de discussion» moralement neutre, d’une manière analogue à celle suivant laquelle ils se différencient historiquement à partir de la «vie éthique substantielle»” (Hegel) ${ }^{38}$.

Se il modello ha un significato solo in quanto dimensione controfattuale di uno stato democratico insufficiente, come ideale che funziona da istanza critica delle pratiche quotidiane, la discussione di questo significato ideale costituisce qui l'aspetto centrale, per non ricadere nell'eternale ritorno della ragione illuministica, nelle illusioni con effetti reali dei proudhoniani di allora e di oggi. Come ricorda il Marx dei Grundrisse, a differenza dei difensori della borghesia, i

\footnotetext{
${ }^{36}$ ROSENFELD, Interpretazioni [2000: 192].

${ }^{37}$ Habermas si interroga sul rapporto tra temporalità storica e modello contrattualistico con riferimento alle generazioni future: "Comment une théorie qui se limite au cercle des destinataires capables de parler et d'agir peut-elle se comporter à l'égard de la vulnérabilité d'une créature muette?”, HABERMAS, De l'éthique de la discussion [1999: 31]. A questa domanda risponde rinviando alla "responsabilità pratica", che però sembra perdere ogni determinazione concreta per il dissolversi della forma etica del discorso nella forma etica del diritto.

${ }^{38}$ APEL, La relation entre morale, droit et démocratie [2001: 68]. Si veda, soprattutto, ID., Penser avec Habermas contre Habermas [1990]. “Or, selon moi, du point de vue de la fondation pragmatique-transcendantale ultime de l'éthique de la discussion [...] il faut que le principe procédural de fondation des normes morales matérielles par la discussion pratique des intéressés ou de leurs mandataires soit lui-même fondé philosophiquement, et précisément par le principe (primordial) de la discussion. Chez Habermas aussi ! Mais alors, le principe procédural ne peut plus être pensé comme dépourvu de toute teneur de sens moral. Il doit inclure a priori l'égalité de droit des intérêts et des arguments possibles de tous les partenaires de la discussion, et aussi à mon sens la coresponsabilité de tous les partenaires de la discussion dans la tentative de résolution consensuelle du problème. Or, ces normes fondamentales transcendantales de l'éthique de la discussion ne peuvent être elles-mêmes un résultat possible de la «discussion pratique», puisque ce sont elles que la rendent d'abord possible. [...]. Ainsi, le principe de discussion n'est en mesure de fonder les normes procédurales de la morale de la discussion que si, et seulement si, comme on peut l'apercevoir grâce à une réflexion transcendantale, il n'est pas luimême moralement neutre, mais contient déjà en manière implicite les normes procédurales fondamentales de la morale de la discussion” (APEL, 2001: 72-73).
} 
nuovi proudhoniani sono coscienti delle contraddizioni inerenti al sistema, ma la loro prospettiva utopistica si manifesta nella incapacità di cogliere la differenza tra forma reale e forma ideale della società borghese, e conseguentemente nel loro desiderio di intraprendere il compito superfluo di voler realizzare l'espressione ideale stessa, allorché in effetti si tratta semplicemente di una immagine fotografica (Lichtbild) della medesima realtà ${ }^{39}$.

Nelle pagine dedicate alla critica della filosofia del diritto, leggiamo infatti che il futuro del popolo tedesco

non può limitarsi né alla immediata negazione delle sue reali condizioni politiche e giuridiche né alla immediata traduzione in pratica di quelle ideali, poiché la immediata negazione delle sue condizioni reali esso la possiede già nelle sue condizioni ideali, e la immediata traduzione in pratica delle sue condizioni ideali a sua volta esso la ha già quasi sopravanzata osservando i popoli suoi vicini ${ }^{40}$.

Il trascendere l'immanenza senza abbandonarla è il momento di verità che solleva l’ideologia borghese al di sopra dell'ideologia stessa, a partire della sfera più intima della vita dei soggetti ${ }^{41}$.

\begin{abstract}
Mentre nella vita borghese l'interesse comune ha un carattere essenzialmente negativo e si concretizza nella difesa contro i pericoli, esso assume un aspetto positivo nell'amore sessuale e innanzi tutto nella cura materna. Lo sviluppo e la felicità dell’altro sono voluti in questa unità [...]. La famiglia borghese conduce così non solo all'autorità borghese, ma anche al presentimento di una migliore condizione umana ${ }^{42}$.
\end{abstract}

Si tratta allora di mettere in questione il modello come significazione immaginaria sociale, cioè il suo fondamento storico-sociale insieme alla produzione che ne deriva. Dimensione così profondamente e autenticamente immaginaria, in un senso reale e positivo, esistente nella realtà proprio in quanto immagine intrinsecamente incastonata in essa, custodita come un segreto necessario alla sua propria struttura, e destinata a rimanere tale, nella storia dell’istituzione immaginaria della società.

\footnotetext{
39 É in gioco, in altri termini, la differenza fondamentale, formulata già ne La questione ebraica, tra emancipazione politica e emancipazione umana. Peraltro l'illusione generata dall'immagine fotografica sembra oggi svanire proprio a causa della convergenza delle razionalità politiche neo-liberale e neo-conservatrice, alla base di una nuova forma di legittimazione, che trova nell'assoggettamento completo alla logica della scelta razionale e nel parallelo ampliamento delle sfere di responsabilità morale e individuale dei cittadini il principio della propria governabilità, come emerge dalle analisi di Wendy Brown sui processi di de-democratization della società americana. BROWN, Neo-liberalism and the end of liberal democracy [2003]; ID., American nightmare: neo-liberalism, neoconservatism and de-democratization [2006]; recentemente raccolti in BROWN, Les habits neufs de la politique mondiale [2007].

${ }^{40}$ MARX, Per la critica della filosofia di Hegel [1991: 57].

41 "I borghesi costituiscono la dimensione pubblica di un dibattito letterario nel quale la soggettività che ha origine nell'intimità della piccola famiglia riesce a raggiungere la comprensione di se stessa", HABERMAS, "Strutture sociali dell'opinione pubblica", in Storia e critica dell'opinione pubblica [2006: 61].

${ }^{42}$ HORKHEIMER, Studi sull'autorità e la famiglia [1976].
} 
L’argomentazione, come medium della comunicazione, come forma riflessiva della comunicazione del mondo della vita, è portavoce della realtà sociale operante, della realtà di campo che sorge a livello di insieme, al di là della dimensione (che Lyotard definisce) molare o molecolare dell'azione dei soggetti. Comunicazione immediata di se stessa, cioè del movimento della medialità che sta al fondo della comunicabilità in generale, l'argomentazione è espressione (non rinvio) dell'alterità immanente alla lingua e che la costituisce come facoltà mimetica del linguaggio ${ }^{43}$, della proprietà di co-esistenza, che Castoriadis definisce nei termini di immaginazione produttiva o creativa, di immaginario radicale o ultimo, in quanto origine comune dell'immaginario effettivo, del simbolico e del razionale.

Le symbolique comporte, presque toujours, une composante «rationnelle-réelle»: ce qui représente le réel, ou ce qui est indispensable pour le penser, ou pour l'agir. Mais cette composante est tissée inextricablement avec la composante imaginaire effective - et cela pose, aussi bien à la théorie de l’histoire qu’à la politique un problème essentiel ${ }^{44}$.

Attraverso la teoria discorsiva del diritto, nel corso dell'argomentazione giuridica si comunica l'immaginario radicale della società, cioè il modo di crearsi e di pensarsi, di definire e di rispondere ai bisogni, di costruirsi in una identità, di una determinata società o epoca storica; creazione incessante e essenzialmente indeterminata (storico-sociale e psichica) di figure/forme/immagini, che è la condizione operante di ogni rappresentazione ulteriore, ma che esiste essa stessa già sul modo della rappresentazione, forse nei termini benjaminiani della somiglianza immateriale, “che fonda le tensioni non solo fra il detto e l'inteso, ma anche fra lo scritto e l'inteso, e altresì fra il detto e lo scritto"45.

Dunque, non creazione di immagini nella mente o nella società, né riflesso e tanto meno immagine speculare di un campo predefinito, ma piuttosto signifiable: "the capacity to posit that which is not, to see in something that which is not there"46.

\footnotetext{
43 "Poiché la lingua non è mai soltanto comunicazione del comunicabile, ma anche simbolo del non-comunicabile", BENJAMIN, Sulla lingua in generale [2006: 69], la facoltà mimetica non riproduce passivamente un mondo già fatto, "ma è quell'originario rapporto col mondo che si fa espressione. Per questo le somiglianze che la facoltà mimetica produce sono immateriali”, MORONCINI, Walter Benjamin e la moralità del moderno [1984: 237].

${ }^{44}$ CASTORIADIS, L'institution imaginaire de la société [1975: 192].

${ }^{45}$ BENJAMIN, Sulla facoltà mimetica [2006: 73]. "Così sarebbe per me dimenticata la camera in cui dormivo a sei anni, se una sera - io ero già a letto - mio padre non vi fosse entrato con la notizia di una morte. In verità non fu la notizia in sé a colpirmi così: il morto era un lontano cugino. Ma nel modo in cui mio padre me lo disse era...", BENIAMIN, Cronaca berlinese [1932, 1997: 267]. "One of the gross inadequacies of Lacan's conception of the imagination is his fixation on the scopic. For me, if one is speaking of stages that are worked out, the imagination par excellence is the imagination of musical composer... suddenly, figures surge forth that are not in the least visual. They are essentially auditory and kinetic - for there is also rhythm”, CASTORIADIS, Logic, imagination, reflection [1995: 182].

${ }^{46}$ CASTORIADIS, Done and to be done [1997].
} 
L’immaginario radicale della comunità ideale di comunicazione - altrove “anticipazioni trascendentali" in quanto "idee regolative" - implica infatti, come ricorda lo stesso Apel, elementi “naturali” o incoscienti della comunicazione linguistica, e include come presupposto trascendentale di ogni critica della ideologia (poiché ne spezza la dinamica totalitaria) il postulato “de una lingüistificación no distorsionada y en principio ilimitada de la competencia extraverbal de la comunicación”47.

Con un doppio movimento - o immagine allo specchio, ma anche dédoublement smascherato da Bourdieu - l'istituzionalizzazione della norma sociale crea l'istituzionalizzazione della società.

Alla normalizzazione della società subentra allora la sua giuridicizzazione: il solo momento della registrazione ${ }^{48}$.

I simboli prendono così l'aspetto dei feticci, come la dodecafonia tanto si avvicina al feticismo del materiale, anche nel rapporto con la serie: "La sonorità, per quanto differenziata, si avvicina a quello che essa era prima che la soggettività ne prendesse possesso: pura e semplice registrazione" 49 .

La violenza simbolica

est une forme de pouvoir qui s'exerce sur les corps, directement, et comme par magie, en dehors de toute contrainte physique.

On ne peut donc penser cette forme particulière de domination qu’à condition de dépasser l'alternative de la contrainte (par des forces) et du consentement (à des raisons), de la coercition mécanique et de la soumission volontaire, libre, délibérée, voire calculée. L’effet de la domination symbolique (qu'elle soit d'ethnie, de genre, de culture, de langue, etc.) s'exerce non dans la logique pure des consciences connaissantes, mais à travers les schèmes de perception, d'appréciation et d'action qui sont constitutifs des habitus et qui fondent, en deçà des décisions de la conscience et des contrôles de la volonté, une relation de connaissance profondément obscure à elle-même. ${ }^{50}$

Tuttavia la suggestione che contiene ancora qualche traccia del terrore che inspira il feticcio non si accorda più alla legittimazione cosciente. Come è stato scritto in maniera efficace e potente nella Dialettica dell'illuminismo, l'unità della collettività e della dominazione - che si esprime nelle categorie del pensiero - si rivela piuttosto nell'universalità che assume necessariamente il contenuto malvagio del reale, sia nella lingua metafisica sia in quella scientifica. In questo processo mimetico,

\footnotetext{
${ }^{47}$ E questo significa la “superación de la mera naturaleza”, APEL, Transformation der Philosophie, vol. 2 [1973: 306]. Citato in SCHELKSHORn, Discurso y liberación, in Dussel, Debate [1994: 31].

${ }^{48}$ Contro la tendenza scientista della comunità ideale di comunicazione, Wellmer parla infatti di un "linguaggio definitivo”, del “sogno originale dell’empirismo logico” e della “completa trasparenza”. WELLMER, Ethik und Dialog [1986].

${ }^{49}$ ADORNO, “Schönberg e il progresso,” in Filosofia della musica moderna [2002: 89].

50 Bourdieu, La domination masculine [2002: 59].
} 
la realtà esistente diviene l'unica realtà, poiché il pensiero reificato - che chiamiamo qui con il nome di norma giuridica - si riduce ad una semplice tautologia ${ }^{51}$.

Reversibilità del dono nel contro-dono, reversibilità del tempo nel ciclo, reversibilità della produzione nella distruzione, reversibilità della vita nella morte, reversibilità di ogni termine e valore linguistico nell'anagramma: un'unica grande forma, la stessa in tutti i campi: quella della reversibilità, della reversione ciclica, dell'annullamento; quella che ovunque mette fine alla linearità del tempo, a quella del linguaggio, a quella degli scambi economici e dell'accumulazione, a quella del potere. Ovunque essa prende per noi la forma della distruzione e della morte. È la forma stessa del simbolico. Né mistica, né strutturale: ineluttabile ${ }^{52}$.

In effetti, lo stesso Habermas, proprio a proposito della istituzionalizzazione dei modelli auto-organizzativi della società, e ammettendo il “controsenso di un’auto-programmata circolazione del potere”, è tornato più volte a raccogliere argomenti

sia contro la cecità sistemica di ogni teoria normativa della democrazia che non si accorga di come i suoi fondamenti siano già stati burocraticamente espropriati, sia contro lo straniamento feticistico di ogni teoria sistemica che liquidi gli elementi normativi ed escluda, fin dal piano dell'analisi, la possibilità di una comunicazione riflessiva della società che si metta a fuoco nel suo insieme ${ }^{53}$.

Se Habermas rifiuta la nozione di un inconscio non-linguistico, come fa anche Lacan, afferma invece la possibilità e il progetto di fare l'inconscio conscio, poiché la repressione dell'inconscio è un esempio intrapsichico di quella che definisce systematically distorted communication $^{54}$. L'inconscio è il campo della significazione privatizzata e distorta, separata e tagliata fuori dalla comunicazione pubblica intersoggettiva. Attribuendo alle forze della repressione l'inibizione dell'autocomprensione del soggetto e dell'azione autonoma collettiva, "the process of autonomy for Habermas entails the elimination of unconscious or desymbolized elements of public communication”55.

\footnotetext{
51 “La relazione simbolica dell’attuale all'evento mitico nel rito o alla categoria astratta nella scienza fa apparire il nuovo come predeterminato, che è così - in realtà - il vecchio. Senza speranza non è la realtà, ma il sapere che - nel simbolo fantastico o matematico - si appropria la realtà come schema e così la perpetua”, HoRKHEIMER - ADORNO, "Concetto di illuminismo", in Dialettica dell'illuminismo [1997: 35].

52 BAUDRILLARD, Lo scambio simbolico e la morte [1976: 12].

53 Habermas, Morale, diritto, politica [2001: 92]. Infatti “è ormai evidente che gli strumenti amministrativi, indispensabili a realizzare i programmi dello stato sociale, non rappresentano affatto un medium passivo e per così dire neutrale", dal momento che "lo stato imprenditore si è a tal punto organizzato a sottosistema indipendente e centrato sul potere, ed ha anche a tal punto espulso nel proprio ambiente i processi della legittimazione, che sembra ormai opportuno formulare diversamente anche l'idea normativa di un'auto-organizzazione della società” (HABERMAS, 2001: 93).

${ }^{54}$ HABERMAS, Knowledge and human interests [1971].

${ }^{55}$ ELLIOT, The social imaginary [2002: 148].
} 
L’autocomprensione libera del soggetto, però, scivola qui lungo due livelli di struttura simbolica del significante ${ }^{56}$, o meglio tre: lungo la superficie fantasmatica che riscrive il reale. La norma giuridica come significazione immaginaria sociale instaura una nuova significazione operante - l'iperreale di Baudrillard -, lasciando Edipo di fronte ad un enigma che resta insoluto (la risposta del soggetto cartesiano come ricerca della propria certezza). Accentuando l'aspetto “passivo” della funzione del soggetto - nell'articolazione significante in cui vi sono tre significanti e non più solo due (l’alienazione lacaniana, infatti, è legata strutturalmente alla funzione della coppia di significanti, perché è nell’intervallo tra questi due significanti che si trova il desiderio offerto nella localizzazione del soggetto nell'esperienza del discorso dell'Altro, ed è proprio in questo intervallo che il soggetto riviene al punto iniziale, che è quello della mancanza come tale, della sua aphanisis $)^{57}-$ Žižek presenta il grand Autre:

Qu'est-ce que la Matrice? Ce n'est rien moins que le «grand Autre», l'ordre symbolique virtuel, le réseau qui structure pour nous la réalité. Cette dimension du grand Autre est celle de l'aliénation constitutive du sujet dans l'ordre symbolique: le grand Autre tire les ficelles, le sujet ne parle pas, c’est la structure symbolique qui parle à travers lui, il «est parlé» par elle ${ }^{58}$.

Ecco allora manifestarsi in una situazione esemplare la matrice simbolica in cui il soggetto si precipita in una forma primordiale.

La traduzione della lingua dell'uomo nel linguaggio muto delle cose (e viceversa), direbbe Benjamin $^{59}$.

\footnotetext{
${ }^{56}$ Questo doppio livello è tuttavia differente da quel doppio livello simbolico che Bourdieu rintracciava nella finzione di uno sdoppiamento del linguaggio in ordinario e specifico, che corrispondeva alle diverse classi sociali (e che, ad esempio, serviva ad Heidegger, Schimtt e Jünger, per indurre il disprezzo dello stato sociale in quanto categoria ontica, a cui si contrappone il Fürsorge dell'ontologia dell'essere, accessibile solo ai filosofi): BouRDIEU, Langage et pouvoir symbolique [2001: 343].

${ }^{57}$ Si veda LACAN, Séminaire XI. Les quatre concepts fondamentaux de la psychanalyse [1964, 1973]. “A savoir, que le signifiant est ce qui représente le sujet pour l'autre signifiant. D’où il résulte qu'au niveau de l'autre signifiant, le sujet s’évanouit”' (LACAN, 1973: 263).

58 ŽIZEK, Matrix, in La subjectivité à venir [2006: 97]. "Mais il est ici capital de se souvenir des chapitres cruciaux du Séminaire XI, où Lacan se force de présenter dans le détail l'opération qui suit l'aliénation et constitue en quelque sorte, son contrepoint, la séparation: l'aliénation Dans le grand Autre est suivie par la séparation Du grand Autre”. Su un altro piano di indagine: “Si l'absorption de l'émancipation dans la régulation [...] neutralisa efficacement les craintes autrefois associées à l'éventualité d'une transformation sociale fondamentale et de futurs alternatifs, produisit un nouveau sentiment d’insécurité provenant de la crainte des développements incontrôlables qui pourraient avoir lieu sur-le-champ, à cause, précisément, de la contingence et du conventionnalisme généralisés des pratiques sociales sectorielles. [...]. D’un point de vue idéologique, la régulation elle-même finit par perdre son crédit en tant que pilier de la modernité, pas, comme par le passé, à cause de son incompatibilité avec l'émancipation, mais, plutôt, à causes de ses contradictions internes. Autrement dit, la contingence globale et le conventionnalisme minent la régulation sans promouvoir l'émancipation. La première devient impossible tandis que la seconde devient impensable”, SousA SANTOS, Vers un nouveau sens commun juridique [2004: 9].

59 “Spazi continui di trasformazione, non astratte regioni di eguaglianza e somiglianza, misura la traduzione”, BENJAMIN, Sulla lingua in generale [2006: 64].
} 
Il processo di reciprocità tra mitologia e Aufklärung, direbbero Horkheimer e Adorno, che fa della ragione la radicalizzazione del terrore mitico e della natura delle cose il substrato della dominazione - a non essere alterato è il principio di equivalenza alla base: se prima i feticci erano sottomessi alla legge dell'equivalenza, ormai è l'equivalenza a essere feticcio ${ }^{60}$.

Il nodo centrale è la relazione tra idee e valori di libertà e di uguaglianza con la logica economica e il sistema del mercato, scrive Jameson ${ }^{61}$, chiedendosi se un concetto così straordinariamente esteso della ragione abbia ancora un valore normativo in un contesto in cui il suo opposto, l’irrazionale, si è ritirato al punto di essere virtualmente inesistente.

In quale senso andremo? Da quale lato tutto si rovescerà, si piegherà o si ripiegherà? Arriva infine, da un altro non-luogo (iper)reale, l'eco deleuziana della lettura-riscrittura di Lewis Carroll ${ }^{62}$.

\section{L'ISTITUZIONE IMMAGINARIA DELLA SOCIETÀ 1968-1999}

Scaturito da una religione del padre, il cristianesimo divenne una religione del figlio. Non sfuggì alla fatalità di doversi sbarazzare del padre.

FREUD, L'uomo Mosé [2006: 452]

Mais, par une étrange malédiction, le politique réussit cette destruction en donnant par exemple naissance à la technostructure ou la bureaucratie, pour lesquelles seule existe l'administration des choses. Reste à voir si, ce faisant, et peutêtre s'agit-il là d'un retour rusé de l'amor fati dénié, il ne prépare pas, en fait, d'une manière inconsciente, sa propre autodestruction.

MAFFESOLI, La transfiguration du politique [1995: 48]

Mais le problème essentiel n'est pas là. Le vrai sort du capitalisme se joue, en effet, face aux hiérarchies sociales.

BRAUDEL, La dynamique du capitalisme [1985: 70]

\footnotetext{
60 "Ma questa dialettica rimane impotente nella misura in cui si sviluppa dal grido di terrore, che è la duplicazione, la tautologia del terrore stesso. Gli dei non possono togliere all'uomo la paura di cui i loro nomi sono l'eco impietrita. L’uomo si illude di essersi liberato dalla paura quando non c'è più nulla di ignoto. Ciò determina il corso della demitizzazione, dell'illuminismo che identifica il vivente col non-vivente come il mito il non-vivente col vivente. L'illuminismo è l'angoscia mitica radicalizzata. La pura immanenza positivistica, che è il suo ultimo prodotto, non è che un tabù per così dire universale. Non ha da esserci più nulla fuori, poiché la semplice idea di un fuori è la fonte genuina dell'angoscia”, HORKHEIMER - ADORNO, “Concetto di illuminismo”, in Dialettica dell’illuminismo [1997: 35].

61 JAMESON, Postmodernismo [2007: 264].

${ }^{62}$ Occorre, infatti, qui preannunciare Deleuze, e come solo il fantasma, in quanto faccia risuonare le due serie insieme, raggiunge tale comprensione, l'evento da comprendere non essendo diverso dalla risonanza stessa: "E come il fantasma riprende tutto su questo nuovo piano dell'evento puro, in questa parte simbolica e sublimata dell'ineffettuabile, attinge anche da tale parte la forza di dirigere l'effettuazione, di duplicarla, di condurne la contro-effettuazione concreta. L'evento infatti si inscrive proprio nella carne, nei corpi, con la volontà e la libertà relative al paziente pensatore, soltanto in virtù della parte incorporea che ne contiene il segreto, cioè il principio, la verità e finalità, la quasi-causa”, DELEUZE, Logica del senso [2007: 194]. Baudrillard: “Il terzo ordine è il nostro; non è più dell’ordine del reale, ma dell’iperreale, e solo là teorie o pratiche, esse stesse fluttuanti e indeterminate, possono coglierlo e colpirlo a morte”, BAUDRILLARD, Lo scambio simbolico e la morte [1976: 13].
} 
Come dice Reich, il sorprendente non è che della gente rubi, che altri facciano sciopero, ma piuttosto che gli affamati non rubino sempre e che gli sfruttati non facciano sempre sciopero.

DELEUZE - GUATTARI, L'anti-Edipo [2002: 32]

Je n'ai jamais cessé en effet de m'étonner devant ce que l'on pourrait appeler le paradoxe de la doxa: le fait que l'ordre du monde tel qu'il est, avec ses sens uniques et ses sens interdits, au sens propre ou au sens figuré, ses obligations et ses sanctions, soit grosso modo respecté, qu'il n’y ait pas davantage des transgressions ou des subversion, de délits et de «folies» [...]; ou, plus surprenante encore, que l'ordre établi, avec ses rapports de dominations, ses droits et ses passe-droits, ses privilèges et ses injustices, se perpétue en définitive aussi facilement, mis à part quelques accidents historiques, et que les conditions d'existence les plus intolérables puissent si souvent apparaître comme acceptables et même naturelles.

BOURDIEU, La domination masculine [1998: 11]

Un soldato tedesco - soltanto uno - non ce la fece al partecipare al massacro. Era il Sonderführer Günter Amon. Più tardi egli testimoniò che, entrato nelle gallerie col suo plotone di esecuzione, vedendo alla luce delle torce gli uomini uccisi svenne: "Rimasi inorridito dallo spettacolo. Uno dei miei camerati prese il mio posto e sparò per me”. Tuttavia, nei più, ogni sensibilità umana era ormai obnubilata se non completamente distrutta. Per quanto possa sembrare terribile, la maggior parte delle vittime cooperò coi massacratori e andò passivamente alla morte.

KATZ, “L'eccidio delle Fosse Ardeatine”, in Morte a Roma [1996: 153]

L’istituzionalizzazione dell’immaginario della società crea una nuova significazione immaginaria, un nuovo modo di farsi della società, che si costruisce attorno a questa significazione immaginaria sociale, strutturante e strutturata, principio organizzativo, significazione centrale, senso e ombra dell’organizzazione sociale.

Ciò di cui però l'individuo è espropriato non è tanto la sua esperienza immaginifica individuale ma quella sociale su cui è fondata la prassi, almeno nella sua forma storica del lavoro.

Se Castoriadis poteva scrivere che l'individuo può produrre solo dei fantasmi privati e non delle istituzioni - perché una significazione sociale immaginaria abbisogna di significanti collettivamente disponibili, ma soprattutto di significati che non esistono sul modo dei significati individuali (cioè come percepiti, pensati o immaginati dal soggetto) -, attraverso la procedura argomentativa, l’istituzione, di cui tutti gli altri elementi sono presenti, è già simbolizzata come tale. L’immaginario sociale nasce orfano, perché i suoi fantasmi sono fantasmi istituzionali.

La norma giuridica, sulla base dell’agire comunicativo - distinta dal registro del razionale, da quello simbolico così come dall'immagine che un popolo può darsi - diviene una significazione centrale, cioè una nuova significazione operante, organizzazione in sistema di significanti e 
significati, che ne permette l'estensione, la moltiplicazione, la modificazione, e di cui l'individuo non ha libera disposizione.

Se il significante acquista un senso per differenziazione (attraverso l'atto con cui viene rubata, per poi essere débruillée, la lettera di Poe nel celebre seminario di Lacan, ad esempio), cioè attraverso l'atto in cui viene sottratto un significato, nella catena delle significazioni, la ripetizione di questa scena primaria determina l'interruzione della catena significante, in un'esperienza di significanti puramente materiali, distinti e irrelati, nei quali termini Lacan definisce la schizofrenia ${ }^{63}$, e parallelamente lo fanno Deleuze e Guattari ${ }^{64}$.

Il principio di organizzazione di una società circoscrive un campo di possibilità. L’introduzione di un nuovo principio di organizzazione comporta un nuovo livello di integrazione sociale. In particolare, determina le strutture in cui sono possibili i cambiamenti istituzionali del sistema, e i limiti dello sviluppo delle forze produttive, in quanto attori. Il coordinamento tra capacità oggettive, rapporti di comunicazione e relazioni di potere, che Habermas chiama di dominazione, comunicazione e attività finalizzata, assume un equilibrio generale, stabilito su di un modello specifico che è quello giuridico.

Costituisce forse il tentativo (del postmodernismo) di dotare il soggetto di una cartografia cognitiva, cioè di una cultura pedagogica e politica in grado di rappresentargli una nuova, accresciuta consapevolezza della sua posizione nel sistema globale? Una nuova institution $d u$ sens ${ }^{65}$, su cui inscrivere il progetto e, prima ancora, la capacità di pensare la totalità, la società nel suo complesso, in un orizzonte di senso comune?

Il fondamentale compito ideologico del nuovo concetto [il postmodernismo] deve nondimeno restare quello di coordinare nuove forme di prassi e abitudini sociali e mentali (in ultima analisi, penso sia questo ciò che aveva in mente Williams con la sua nozione di "struttura del sentimento”) con le nuove forme della produzione e dell'organizzazione economica portate alla luce dal mutare del capitalismo negli ultimi anni, cioè la nuova divisione globale del lavoro ${ }^{66}$.

Però,

el sistema capitalista es mundial. Hay una estructura de circulación del valor en todo el mundo. En los análisis de la Teoría de la Acción Comunicativa no se considera este doble aspecto: se toma la "parte” (capitalismo central, llamado "tardío” ideologícamente) por el "todo" (el sistema mundial), con lo que se oculta, primero, la función de dominación del "centro" capitalista, y, segundo, no puede explicarse de ninguna manera la pobreza, miseria

\footnotetext{
${ }^{63}$ LACAN, Le séminaire sur «La lettre volée» [1966: 11]. Oltre al celebre De la psychose paranoïaque dans ses rapports avec la personnalité, in cui la paranoia è una forma di conoscenza mediata dell'Io [1932, 1975].

${ }^{64}$ Deleuze, GuATtARI, L’Anti-CEdipe [1972].

${ }^{65}$ DESCOMBES, Les Institutions du sens [1996]; TAYLOR, Il disagio della modernità [1998].

66 JAMESON, Postmodernismo [2007: 10].
} 
del capitalismo “periférico” (que debería ser ahora comprendido como “explotado”). Pareciera que es una cuestión secondaria, pero produce la implantación ideológica de toda la estructura categorial, y por ello invalida tanto el "sentido" de lo analizado en el "centro" como en la "periferia". A esto hemos llamado la "falacia del desarrollismo" (Felschluss der Entwicklung-sideologie), aspecto del "eurocentrismo” (que invalida en parte la filosofia habermasiana y la teología que usa sus categorías) ${ }^{67}$.

E soprattutto,

independizada la economía del "mundo de la vida cotidiana" (Lebenswelt), viene a yuxtaponerse como un "sistema". En ese caso, la cultura, la religión, constituyen momentos de dicha Lebenswelt, y en relación con ésta la teología efectúa su reflexión. De todas maneras, no podrá evadirse del estrecho horizonte de la "comunidad de comunicación" donde se cumple la "acción comunicativa"68.

Il codice giuridico riempie allora lo scarto tra l'esperienza esistenziale e la conoscenza scientifica, implicito nella ridefinizione althusseriana (e lacaniana) dell’ideologia come rappresentazione del rapporto immaginario degli individui con le proprie condizioni di vita ${ }^{69}$, e riveste la funzione di inventare in qualche misura una modalità di articolazione tra queste due dimensioni distinte.

Il codice giuridico non è soltanto collegato con il medium del linguaggio ordinario, cioè con il medium attraverso cui scorrono le prestazioni d'intesa socio-integrative del mondo di vita. Esso può anche tradurre i messaggi che nascono dal mondo di vita rendendoli comprensibili ai codici speciali dell'amministrazione e del denaro. In questo senso il linguaggio del diritto - diversamente dalla comunicazione morale che si limita alla sfera del mondo di vita - può funzionare da trasformatore entro quella circolazione comunicativa che, attraverso tutto il corpo sociale, viene ad instaurarsi tra "sistema" da un lato e "mondo di vita" dall’altro".

La conclusione (circolare) è che "il diritto funziona così da trasformatore: esso solo è in grado di garantire che non si spezzi la rete della comunicazione generale che tiene insieme tutta la società. Solo nel linguaggio del diritto possono circolare per tutto il corpo sociale messaggi ricchi di contenuto normativo"71.

Così i blocchi foucaultiani di capacité-communication-pouvoir, estensioni della società civile egemonica del Gramsci dei Quaderni - dalla scuola, alla famiglia, agli ospedali e istituzioni psichiatriche, alle carceri, fino ad arrivare al corpo - sono espressione di un grande sistema, regolato e concertato, di una forma sociale ${ }^{72}$.

\footnotetext{
${ }^{67}$ Dussel, Les metáforas teologícas de Marx [1993: 264-265].

${ }^{68}$ DusSEL, Les metáforas teologícas de Marx [1993: 277].

${ }^{69}$ Althusser, Idéologies et appareils idéologiques d'État [1976].

${ }^{70}$ HABERMAS, Fatti e Norme [1996: 101].

${ }^{71}$ HABERMAS, Fatti e Norme [1996: 72]. Come ci ricorda Foucault, ciò che definisce una relazione di potere è une action sur des actions. Possiamo collocare qui una differenziazione tra Atto e Azione, che è stata analizzata da Jacques Lacan nei suoi ben noti seminari, in particolare ne Les quatre concepts fondamentaux de la psychanalyse [1964, 1973].

${ }^{72} \mathrm{O}$, in termini leniniani, di una "formazione sociale determinata".
} 
Così la vera esistenza democratica, l'esistenza umana, si afferma, per essere colta, solo in quanto esistenza giuridico-democratica. Tuttavia, l'esistenza soppressa nel diritto, negata e conservata - come ci insegna il giovane Marx della Critica della dialettica hegeliana - per essere revocata e riportata al soggetto, è già esistenza pensata, alienata ${ }^{73}$.

Come nell’ulteriore capovolgimento della matrice edipica, guardando più da vicino la scena della messa a morte, che non può non avere il carattere pubblico dell'esecuzione, possiamo anche annotare che

en lieu et place de l'obscène père présymbolique primordial qui revient après son meurtre sous l'aspect du Nom, de l'autorité symbolique, nous avons à présent l'autorité symbolique (logos) trahie, mise à mort par ses successeurs/fils, puis revenant sous l'aspect de la figure jalouse et impitoyable du surmoi, celle du Dieu rempli de rage meurtrière ${ }^{74}$.

Per riprendere qui una nota critica ad Habermas, brillantemente risolta da Foucault, come vedremo nel paragrafo successivo, la messa a morte democratica del patriarca non è questione che possa risolversi su un piano soltanto procedurale. Non sono i tanti che agiscono legittimamente per mano dei singoli delegati, né la bocca dell’uno che parla a nome della moltitudine, ma si tratta del volto proprio del potere, nella forma del comando, della legge, del processo, della sentenza, o nella forma della violenza e dell'esecuzione ${ }^{75}$.

\section{OMNES ET SINGULATIM 2000}

Just call it.

I didnt put nothing up.

Yes you did. You've putting it up your whole life. You just didnt know it. You know what the date is on this coin?

No.

It's nineteen fifty-eight. It's been travelling twenty-two years to get there. And now it's here. And I'm here. And I've got my

\footnotetext{
73 "Parimenti la qualità soppressa è uguale a quantità, la quantità soppressa è uguale a misura, la misura soppressa è uguale a essenza, l'essenza soppressa è uguale a fenomeno, il fenomeno soppresso è uguale a realtà, la realtà soppressa è uguale a concetto, il concetto soppresso è uguale ad oggettività, l'oggettività soppressa è uguale a idea assoluta, l'idea assoluta soppressa è uguale a natura. La natura soppressa è uguale a spirito soggettivo, lo spirito soggettivo soppresso uguale a spirito soggettivo etico, lo spirito etico soppresso è uguale ad arte, l'arte soppressa è uguale a religione, la religione soppressa è uguale a sapere assoluto”, MARX, Manoscritti economico-filosofici [2004: 169].

74 ŽIZEK, Le sujet qui fâche [2007: 422]. Applichiamo anche al recente e sottovalutato lavoro cinematografico The Assassination of Jesse James by the Coward Robert Ford (DOMINIK, USA 2007) l'intuizione di Zizek per cui “ce que le successeur fidèle de la figure paternelle du Leader est susceptible de cacher, c'est précisément cet écart entre le Leader dans l’immédiateté de sa personnalité et le lieu symbolique qu'il occupe, cet écart en vertu duquel le père comme personne effective est ouvertement impuissante et ridicule” (ŽIZEK, 2007: 419).

${ }^{75}$ Non a caso, nel bellissimo film The last temptation of Christ (Usa, 1988), Scorsese dedica pochissime sequenze al processo di Cristo, solo apparentemente depoliticizzandolo, e in effetti radicalizzandolo. Sullo "scandalo intellettuale" della pena e sul processo come quadro della ripetizione simbolica della scena effettiva della violenza, si vedano le analisi di Paul RiceEur, Le juste, la justice et son échec [2006], e Le juste 2 [2001], e di Otfried HöFFE, "La facoltà di punire: elementi costruttivi di una teoria”, in HÖFFE, Globalizzazione e diritto penale [2001: 79].
} 
hand over it. And it's either heads or tails. And you have to say.

Call it.

I dont know what it is I stand to win.

In the blue light the man's face was beaded thinly with sweat.

He licked his upper lip.

You stand to win everything, Chigurh said. Everything.

You aint making any sense, mister.

Call it.

Heads then.

Chigurh uncovered the coin. He turned his arm slightly for the man to see. Well done, he said.

MCCARTHY, No country for old men [2005: 56]

Humain, il serait coupable, et, mythique, il ne cesse pas de l'être: il reste souillé de son acte, et la purification, si elle est nécessaire, n’est jamais complète.

CAILLOIS, Le mythe et l'homme [1938: 28]

Dr F - then intimated a desire to have me put a question. I did so, as follows:

"M. Valdemar, can you explain to us what are you feelings or wishes now?"

There was an instant return of the hectic circles on the cheeks: the tongue quivered, or rather rolled violently in the mouth (although the jaws and lips remained rigid as before), and at length the same hideous voice which I have already described, broke forth:

"For God's sake! - quick! - quick ! - put me to sleep - or, quick ! - waken me! - quick! - I say to you that I am dead!”

POE, The facts in the case of M. Valdemar, in Selected tales [1994: 373]

La procedura è la forma storica che assume l'immaginario sociale e l'istituzionalizzazione dell’immaginario sociale la fase attuale dell'istituzione immaginaria della società, che corrisponde alla fase più elaborata della razionalizzazione del pensiero.

La société est donc toujours auto-institution du social-historique. Mais cette auto-institution généralement ne se sait pas comme telle (ce qui a fait croire qu'elle ne peut pas se savoir comme telle). L’aliénation ou hétéronomie est auto-aliénation; occultation de l'être de la société comme auto-institution à ses propres yeux, recouvrement de sa temporalité essentielle. Cette auto-aliénation [...] se manifeste dans la représentation sociale (elle-même, chaque fois, instituée) d'une origine extra-sociale de l'institution de la société (origine imputée à des êtres surnaturels, à Dieu, à la nature, à la raison, à la nécessité, aux lois de l'histoire ou à l'être-ainsi de l'Etre). À ce point de vue, une part essentielle de la pensée héritée n’est que rationalisation de cette hétéronomie de la société et, comme telle, une de ses manifestations. Ses réponses à la question du monde et de l'histoire se situent toujours sur un terrain d'où sont, par construction, exclus l'imaginaire radical comme social-historique et comme imagination radicale, l’indétermination, la création, la temporalité comme altération essentielle ${ }^{76}$.

\footnotetext{
${ }^{76}$ CASTORIADIS, L'institution imaginaire de la société [1975: 537]. "Bien évidemment, l'auto-aliénation ou hétéronomie de la société n’est pas «simple représentation» ou incapacité de la société de se représenter autrement que comme instituée depuis et par ailleurs. Elle est incarnée, fortement et lourdement matérialisée dans l’institution concrète de la société, incorporée dans sa division conflictuelle, portée et médiatisée par toute son organisation, interminablement reproduite dans et par le fonctionnement social, l'être-ainsi des objets, des activités, des individus sociaux".
} 
Il momento di affermazione delle soggettività nella produzione normativa corrisponde, dunque, al momento in cui l'uomo aliena se stesso, poiché trasferisce se stesso in qualcosa che è di sua produzione, ma che si autonomizza e diventa altro da sé.

L'immaginario uscito dalla rivoluzione di febbraio del 1848 è sintetizzato da una parola: fraternité. Nell'idea dei proletari, dunque, i quali scambiavano l'aristocrazia finanziaria con la borghesia in generale; nell'immaginazione dei valentuomini repubblicani, i quali negavano l'esistenza stessa delle classi [...], nelle frasi ipocrite delle frazioni borghesi fino ad allora escluse dal potere, il dominio della borghesia era stato soppresso con la proclamazione della repubblica. Tutti i monarchici si trasformarono in repubblicani e tutti i milionari di Parigi in operai. La frase che corrisponde a questa pretesa eliminazione dei rapporti di classe fu fraternité [...] Questa idillica astrazione dai contrasti di classe [...], questo immaginario elevarsi al di sopra della lotta di classe [...]. Ciò che divideva le classi era un semplice malinteso, e Lamartine il 24 febbraio battezzò il governo provvisorio: "un governo che sospende questo terribile malinteso che esiste... tra le diverse classi”77.

Nella procedura giuridico-argomentativa gli individui garantiscono il meccanismo attraverso cui il significato sopravvive alle istituzioni, determinando l'autonomia e la dominanza del momento immaginario nell'istituzione, e dunque dell'istituzione relativamente alla società - senza riconoscere nell'immaginario delle istituzioni il proprio prodotto, poiché la società vive i suoi rapporti con le sue istituzioni sul modo dell'immaginario (si legga: contrainte juridique). “L’appropriazione si presenta come estraniazione, come alienazione, e l'alienazione come appropriazione, la condizione di straniero come la vera cittadinanza"78.

Quanto più il modello è realizzato nelle sue presupposizioni ideali, trasferite nella produzione giuridica, tanto più si assiste allo sradicamento dalla prassi dell'immaginario sociale. Il modello realizzato, che abbracci la comunità interpretativa idealmente allargata ed illimitata, traspone il momento della validità, l'estende come un attimo eterno: il momento dell’idealità si incarna, nella razionalità.

Non è dunque la fattività come tensione interna alla norma a scomparire, ma la dimensione sociale come realtà autonoma e specifica, e non solo come segmento di realtà nella struttura della dimensione "giuridico-sociale”, produttiva di prassi e pratiche politiche nuove. Annullando ogni tensione esterna viene istituzionalizzata l'alienazione. Questa tensione giuridico-normativa produrrà, infatti, nuove forme e specifiche relazioni di potere sulla base dell'autodisciplina come immaginario sociale, legato in maniera irreversibile alla propria identità dalla coscienza e dalla conoscenza di sé (che assumono ora statuto e forma di norma giuridica e di razionalità).

\footnotetext{
${ }^{77}$ MARX, Le lotte di classe in Francia [2003].

${ }^{78}$ MARX, Manoscritti economico-filosofici del 1844 [2004: 81].
} 
La procedura è affermazione, gesto incondizionato dell’indifferenziato. Comprendere l’insignificanza dell’immaginario nella catena delle significazioni si fa allora più difficile, e più difficile è creare una forma di resistenza. L'immaginario sociale è codificato e rinforzato dallo strumentario del diritto. Ciò che ne deriva è il rafforzamento dell'idea della necessità del diritto, e l'impossibile immaginazione (come elemento rigenerante del processo infinito di liberazione, secondo una linea teorica che va da Spinoza, Marx, Benjamin...) di una diversa relazione tra gli uomini.

Il processo delineato dalle analisi foucaultiane - il passaggio del tipo di potere pastorale dalle istituzioni cristiane a quella statale, le développement de la "technologie pastorale" dans la direction des hommes ${ }^{79}$ - si è compiuto: gli individui consacrano se stessi al diritto e vi trasferiscono il tessuto che li costruisce ed agisce, e che contemporaneamente costruiscono ed agiscono.

L’idea della democrazia procedurale può essere letta in controluce come una nuova forma di organizzazione del potere pastorale, la forma più pura e completa, senza interferenza e perturbazioni: quella, non più solo religiosa e non più oligopolio dello stato, primitiva, della società. Nei termini, forse, di quella оıкоvо $\mu \iota \alpha$ in quanto governamentalità su cui si soffermava Agamben in alcuni recenti seminari parigini ${ }^{80}$.

La teoria discorsiva del diritto, di cui il cuore è la tecnica argomentativa, strategia di saperi, è l'espressione di una forma rinnovata di potere costante, individuale e finale, dove la sacralità del postmoderno è sintetizzata dal silenzio della norma giuridica ${ }^{81}$.

Gli uomini sono sovrani di se stessi e degli altri, in quanto pastori della norma giuridica.

L’umanità diviene lo stato che esercita il potere pastorale.

La dominanza si esercita sulla totalità, cioè su stessa, poiché ogni autogoverno, ci insegna Reich, è un’autodittatura ${ }^{82}$.

\footnotetext{
${ }^{79}$ FOUCAULT, «Omnes et singulatim»: vers une critique de la raison politique [2001: 953].

${ }^{80}$ Nel gennaio 2008, nell’Anfiteatro 45b dell’Université Paris 6, Pierre et Marie Curie, il filosofo italiano ha ricostruito la genealogia teologica del pensiero economico e del governo, a partire dalle ricerche contenute nel suo ultimo lavoro, AgAmben, Il Regno e la Gloria [2007]. Nelle pagine finali della traduzione francese l'autore così conclude: "La modernité, ayant fait disparaître Dieu du monde, non seulement n’est pas sortie de la théologie, mais n'a fait, en somme, qu'accomplir le projet de l'oikonomia providentielle” (AGAMBEN, Le Règne et la gloire, 2008).

${ }^{81}$ Come quel silenzio della Natura della Sottile linea rossa di Terrence Malick (MALICK, The thin red line, USA 1998). Perché non intendere, infatti, sulle tracce del grande regista di Austin, la Natura come un sistema di "forme comunicative senza soggetto” (HABERMAS, 2001: 97), paradossalmente così funzionali alla violenza e alla sua incarnazione storica più ricorsiva: la guerra?

${ }^{82}$ ReICH, Psicologia di massa del fascismo [2002]. Si consideri anche la lettura del testo hegeliano della Fenomenologia, in particolare della lotta delle autocoscienze per il riconoscimento e della figura della Servitù-signoria, svolta da Moroncini a partire della crisi della categoria di sovranità politica e della sua sostituzione con i concetti considerati equivalenti di amministrazione e di governo. Per l'autore, infatti (sulla base della griglia interpretativa fornita da due modelli di analisi, Foucault di Il faut défendre la société e Lacan del seminario sull'etica della psicoanalisi, ma, ovviamente, Freud della pulsione di morte), la verità del rapporto servo-padrone non è dovuta "alla ribellione del servo
} 
Se il pastore è tutta la società, cioè ogni singolo individuo, si realizzano i giorni felici in cui l’umanità è governata direttamente da dio, dal verbo che diventa carne, dal logos, dalla legge.

Le pasteur chrétien agit dans une économie subtile du mérite et du démérite, une économie qui suppose une analyse en éléments ponctuels, des mécanismes de transfert, des procédures d'inversion, des jeux d'appui entre éléments contraires, bref toute une économie détaillée des mérites et des démérites entre lesquelles, finalement, Dieu décidera.

Car c’est là aussi un élément fondamental: c'est que finalement, cette économie des mérites e des démérites que le pasteur a à gérer sans arrêt, cette économie n’assure absolument pas de façon certaine et définitive le salut ni du pasteur ni des brebis ${ }^{83}$.

E cioè, lanciando in aria la monetina da due volti, nell'allegoria che va dal Mosé d’Egitto al Mosé di Michelangelo, e da Anton Chigurh (di Bardem) allo sceriffo (Tommy Lee Jones), nell’ultimo deserto attraversato dai Coen e da McCarthy ${ }^{84}$, con la scomparsa dell'efficienza simbolica, entriamo - come nel passamano di soldi sporchi - nella tragedia moderna che segna la differenza tra tirannia e terrore, per cui Lacan può chiamare questo Dio dell’Interdetto le père réel.

En ce sens, la terreur n'est pas un pouvoir de corruption qui mine l'attitude éthique de l'extérieur. Elle la mine plutôt de l'intérieur, mobilisant et exploitant jusqu’à son extrémité l'écart inhérent au projet éthique lui-même, l'écart qui sépare la Cause éthique réelle de la Cause dans sa dimension symbolique (valeurs, etc.), ou, pour le poser en termes politicojuridiques, l'écart qui sépare le Dieu du pur acte de décision du Dieu des Interdits et des Commandements positifs ${ }^{85}$.

Nel lancio della monetina vediamo ormai spezzarsi e disperdersi la traiettoria - resa già invisibile ai nostri occhi - che dalla produzione porta alla circolazione, che dal lavoratore porta al mercato, che dal lavoro vivo porta al prezzo. “Il distacco del soggetto dall'oggetto, premessa dell'astrazione, è fondato nel distacco dalla cosa, a cui il padrone perviene mediante il servitore"86.

Le disuguaglianze sono ora frutto del caso: il puramente irrazionale e contraddittorio con la ragione, in un procedimento analogo alle composizioni dodecafoniche di Schönberg: ogni suono in tutta la composizione è determinato da questa "serie" o "figura fondamentale”, da intendere come un ordinamento determinato di volta in volta dei dodici suoni disponibili nel sistema temperato, per cui,

contro il suo signore o alla potenza emancipativa del lavoro, ma soltanto ad un processo che potremmo definire di interiorizzazione del comando: è perché la coscienza servile accoglie in sé quella signorile, vi si identifica, la riconosce come parte o momento di se stessa, parte o momento necessari alla sua costituzione, che il signore in carne ed ossa può eclissarsi, abbandonare lo spazio esterno della storia e della società ed installarsi in quello interno e privato della psiche”, MORONCINI, Requiem per la scomparsa del padrone [2005: 107-108].

${ }^{83}$ FOUCAULT, Sécurité, territoire, population [2004: 176].

${ }^{84}$ No country for old man (J. e E. COEN, USA 2007). Su cui MALAUSA, Le grand retour [2008: 20].

85 ŽIZEK, Le sujet qui fâche [2007: 427]. In un senso simile, la nozione di "présent autarcique”, che "corrode l'espace symbolique et réel de la distance”, in LAÏDI, Le sacre du présent [2000: 118].

${ }^{86}$ HorkHEIMER - AdORnO, “Concetto di illuminismo”, in Dialettica dell'illuminismo [1997: 21]. 
come nota Adorno, il procedimento variativo viene retrocesso nel materiale, e lo preforma prima che incominci la composizione propriamente detta ${ }^{87}$. Non esistono più note libere:

Il libero gioco di forze della musica tradizionale, che produce l'insieme da un accordo all'altro senza che l'insieme fosse già premeditato di accordo in accordo, è sostituito con l' «inserzione» degli accordi estranei tra loro. Non c'è più un'anarchica attrazione reciproca tra i suoni, ma resta solo la loro monadica mancanza di relazioni e l'autorità pianificatrice che li domina tutti: unico risultato possibile, il caso ${ }^{88}$.

L’uomo dona la sua voce al diritto ${ }^{89}$.

La procedura è la reificazione del discorso dell'Altro (du grand Autre).

L’immaginario radicale, nel suo movimento contrario, la lettre volée (o l’ultima tentazione di Cristo).

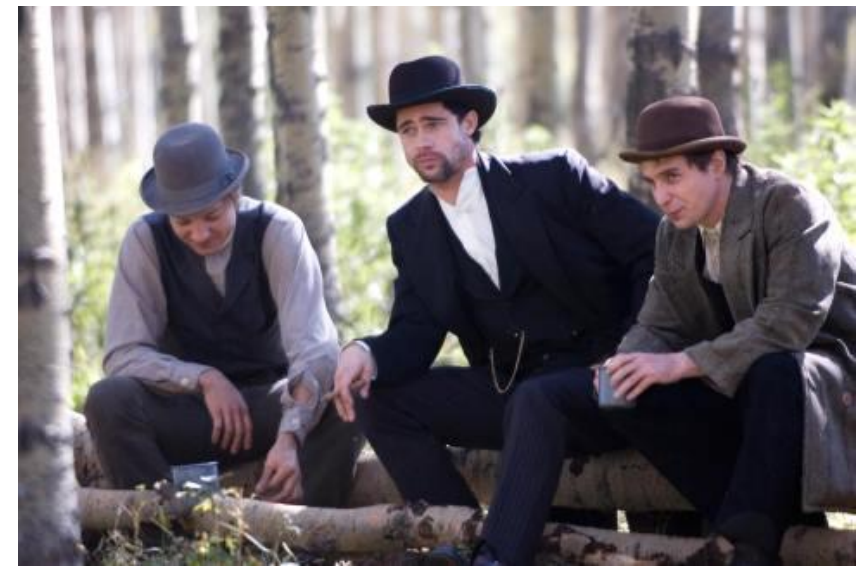

DOMINIK, The Assassination of Jesse James by the Coward

Robert Ford (Usa, 2007)

\begin{abstract}
${ }^{87}$ ADORNO, “Schönberg e il progresso”, in Filosofia della musica moderna [2002: 63].
${ }^{88}$ ADORNO, "Schönberg e il progresso", in Filosofia della musica moderna [2002: 85]. "L'aspetto restaurativo della dodecafonia si dimostra con violenza forse più che altrove nell'allentamento della proibizione della consonanza. Si potrebbe certo dire che l'universalità della dissonanza ne ha superato il concetto stesso, che solo nella tensione con la consonanza la dissonanza è possibile, e che questa si muta in un semplice complesso di suoni appena non si trovi più contrapposta alla consonanza. Tutto ciò però semplifica i nessi reali. Nell'accordo infatti la dissonanza è superata unicamente nel doppio senso hegeliano. I nuovi accordi non sono gli accordi successori delle vecchie consonanze, ma se ne differenziano in quanto la loro unità è totalmente articolata in sé: i singoli suoni dell'accordo si uniscono per conformarlo, ma nel suono interno essi vengono contemporaneamente distinti l'uno dall'altro come suoni singoli. Così continuano a «dissonare»: e non rispetto alle consonanze eliminate, ma in se stessi. In questo modo però tengon salda l'immagine storica della dissonanza" (ADORNO, 2002: 86).

89 “Elle n'est qu'un geste négatif qui donne son expression à la colère malicieuse et vengeresse de Dieu (toute la signification est déjà présente dans l'ordre symbolique qui structure notre univers), mais c'est précisément en tant que telle qu'elle rend effective la signification purement structurelle, et la transforme en une expérience du Sens”, ŽızEK, Le sujet qui fâche [2007: 424]. In altri termini, attraverso la voce che manifesta la Sua Volontà, questo Dio si soggettivizza. Si veda il Seminario inedito di Jacques Lacan sull’Angoscia (1960-1961).
\end{abstract}




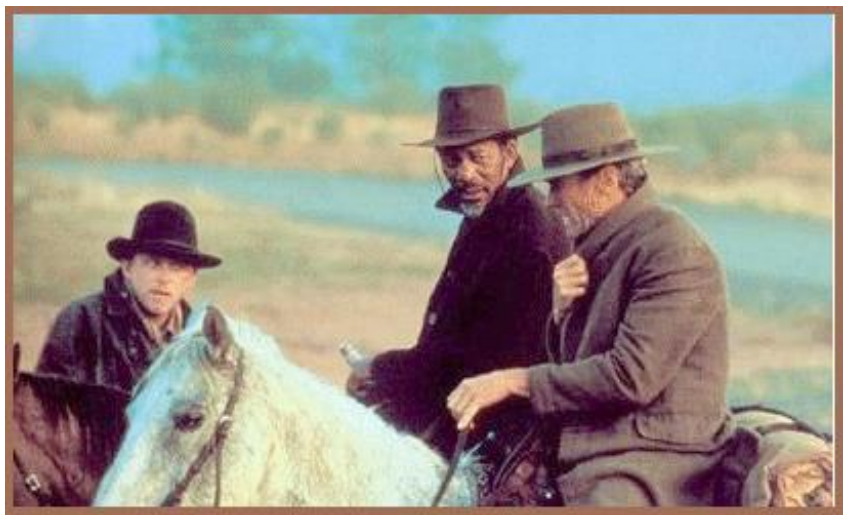

EASTWOOD, Unforgiven (Usa, 1992)

I dont know if law enforcement work is more dangerous now than what it used to be or not. I know when I first took office you'd have a fistfight somewheres and you'd go to break it up and they'd offer to fight you. And sometimes you had to accommodate em. They wouldn't have it no other way. And you'd better not lose, neither.

MCCARTHY, No country for old men [2005: 38]

\section{LETTURE}

ADORNO, Theodor W. [2002] - Filosofia della musica moderna, Torino, Einaudi.

AGAMBEN, Giorgio [2007] - Il Regno e la Gloria. Per una genealogia teologica dell'economica e del governo, Milano, Neri Pozza.

ALTHUSSER, Louis [1965, 2005] - Pour Marx, Paris, La Découverte.

ALTHUSSER, Louis [1976] - Idéologies et appareils idéologiques d'État, in Positions, Paris, Ed. Sociales.

APEL, Karl Otto - DUSSEL, Enrique [1999] - Etica della comunicazione ed etica della liberazione, a cura di A. Savignano, Napoli, Editoriale scientifica.

APEL, Karl Otto [1973] - Transformation der Philosophie, vol. 2, Frankfurt, Suhrkamp.

APEL, Karl Otto [1990] - Penser avec Habermas contre Habermas, Paris, Éditon de l’Éclat.

APEL, Karl Otto [1994] - Fundamentos de semiótica: sentido lingüístico e intencionalidad. La compatibilidad del "giro lingüístico" y el "giro pragmático" de la teoría de la semiótica trascendental, in Dussel, Debate en torno a la ética del discurso di Apel. Diálogo filosófico NorteSur desde América Latina, Mexico-Madrid, Siglo veintiuno editores, 254-312.

APEL, Karl Otto [2001] - La relation entre morale, droit et démocratie. La philosophie du droit de Jürgen Habermas jugée du point de vue d'une pratique transcendantale, in Les études philosophiques, 2001/1, n. 56, 67-80.

BAUDRILLARD, Jean [1976, 2007] - Lo scambio simbolico e la morte, Milano, Feltrinelli. 
BENJAMIN, Walter [2006] - Sulla facoltà mimetica, in Angelus Novus. Saggi e frammenti, a cura di R. Solmi, con un saggio di F. Desideri, Torino, Einaudi, 71.

BENJAMIN, Walter [2006] - Sulla lingua in generale e sulla lingua dell'uomo, in Angelus Novus. Saggi e frammenti, a cura di R. Solmi, con un saggio di F. Desideri, Torino, Einaudi, 53.

BORCH-JACOBSEN, Mikkel [1995] - Lacan. Le maître absolu, Paris, Flammarion.

BOURDIEU, Pierre [1998] - La domination masculine, Paris, Seuil.

BOURDIEU, Pierre [2001] - Langage et pouvoir symbolique, Paris, Fayard.

BROWN, Wendy [2001] - Politics out of history, Princeton univ. pr.

BROWN, Wendy [2003] - Neo-liberalism and the end of liberal democracy, in Theory \& Event, 2003, vol. 7, n. 1.

BROWN, Wendy [2006] - American nightmare: neo-liberalism, neoconservatism and dedemocratization, in Political theory, 2006, 34.

BROWN, Wendy [2007] - Les habits neufs de la politique mondiale. Néolibéralisme et néoconservatisme, Paris, Les prairies ordinaires.

CASTORIADIS, Cornelius [1975] - L’institution imaginaire de la société, Paris, Seuil.

CASTORIADIS, Cornelius [1995] - Logic, imagination, reflection, in Elliott - Frosh (dir.), Psychoanalysis in contexts: paths between theory and modern culture, London, Routledge.

CASTORIADIS, Cornelius [1997] - Done and to be done, in Curtis - Ames, The Castoriadis reader, Oxford, Blackwell.

DELEUZE, Gilles - GUATTARI, Felix [1972] - L'Anti-(Edipe, Paris, Minuit (trad. italiana di Alessandro Fontana, L'anti-Edipo. Capitalismo e schizofrenia, Torino, Einaudi, 2002).

DELEUZE, Gilles [1967] - Présentation de Sacher-Masoch. Le Froid et le Cruel, Paris, Minuit.

DELEUZE, Gilles [1969] - Logique du sens, Paris, Minuit (trad. italiana di Mario De Stefanis, Logica del senso, Milano, Feltrinelli 2007).

DELEUZE, Gilles [1970] - Spinoza, Paris, PUF.

DERRIDA, Jacques [1994] - Force de loi, Paris, Galilée.

DESCOMBES, Vincent [1996] - Les Institutions du sens, Paris, Minuit.

DIAMOND, Elin [2004] - Feminst readings of Beckett, in Oppenheim, L. [ed. 2004] - Samuel Beckett studies, London, Macmillan (Palgrave).

DUSSEL, Enrique [1977] - Filosofía de la Liberación, México, Edicol.

DUSSEL, Enrique [1993] - Las metáforas teologícas de Marx, Estella, edv. 
DUSSEL, Enrique [1994] - Debate en torno a la ética del discurso de Appel. Diálogo filosófico Norte-Sur desde América Latina, Mexico-Madrid, Siglo veintiuno editores.

DUSSEL, Enrique [1999] - Sei principi dell'architettonica dell'Etica della liberazione, in Apel Dussel, Etica della comunicazione ed etica della liberazione, a cura di A. Savignano, Napoli, Editoriale scientifica.

FOUCAULT, Michel [1976] - Sorvegliare e punire. Nascita della prigione, Torino, Einaudi.

FOUCAULT, Michel [2000] - Io, Pierre Rivière, avendo sgozzato mia madre, mia sorella e mio fratello... Un caso di parricidio nel XIX secolo a cura di Michel Foucault, introduzione di Paolo Crepet, Torino, Einaudi.

FOUCAULT, Michel [2001] - «Omnes et singulatim»: vers une critique de la raison politique, in Dits et écrits II, 1976-1988, Paris, Gallimard, 953.

FOUCAULT, Michel [2004] - Sécurité, territoire, population. Cours au Collège de France. 19771978, Paris, Seuil/Gallimard.

FREUD, Sigmund [2006] - L'uomo Mosé e la religione monoteistica e altri scritti, in Opere 1930 1938, Torino, Bollati Boringhieri.

HABERMAS, Jürgen [1971] - Knowledge and human interests, trad. Jeremy J. Shapiro, Boston, Beacon Press.

HABERMAS, Jürgen [1987] - "Excursus on Cornelius Castoriadis: the imaginary institution”, in The philosophical discourse of modernity, trad. Frederick Lawrence, Cambrudge, Polity, 327-335.

HABERMAS, Jürgen [1996] - Fatti e Norme. Contributi a una teoria discorsiva del diritto e della democrazia, a cura di L. Ceppa, Milano, Guerini e Associati.

HABERMAS, Jürgen [1999] - De l’éthique de la discussion, Paris, Flammarion/Champs.

HABERMAS, Jürgen [2001] - Morale, diritto, politica, Torino, Edizioni di Comunità.

HABERMAS, Jürgen [2006] - Storia e critica dell’opinione pubblica, Roma-Bari, Laterza.

HÖFFE, Otfried [2001] - Globalizzazione e diritto penale, Torino, Edizioni di Comunità.

HORKHEIMER, Max - ADORNO, Theodor W. [1947, 1997] - Dialettica dell'illuminismo, Torino, Einaudi.

HORKHEIMER, Max [1976] - Studi sull’autorità e la famiglia, Torino, UTET.

JAMESON, Friedric [2007] - Postmodernismo ovvero La logica culturale del tardo capitalismo, Roma, Fazi.

KLOSSOWSKI, Pierre [1933] - Eléments d'une étude psychanaytique sur le marquis de Sade, in Revue de Psychanalyse. 
LACAN, Jacques [1932, 1975] - De la psychose paranoïaque dans ses rapports avec la personnalité suivi de Premiers écrits sur la paranoïa, Paris, Seuil.

LACAN, Jacques [1964, 1973] - Les quatre concepts fondamentaux de la psychanalyse. Séminaire, Livre XI, texte établi par J.-A. Miller, Paris, Seuil.

LACAN, Jacques [1966, 1999] - Écrits, Paris, Seuil.

LACAN, Jacques [2005] - Introduction aux noms-du-Père, in Des noms-du-Père, Paris, Seuil, 67.

LAÏDI, Zaki [2000] - Le sacre du présent, Paris, Champs/Flammarion.

LEGENDRE, Pierre [2000] - Le crime du caporal Lortie. Traité sur le Père, Paris, Flammarion, Champs.

LUHMANN, Niklas [1995] - Procedimenti giuridici e legittimazione sociale, Milano, Giuffrè.

LUHMANN, Niklas [2002] - I diritti fondamentali come istituzione, Bari, Dedalo.

LYOTARD, Jean-François [1979] - La condition postmoderne. Rapport sur le savoir, Paris, Minuit.

MARX, Karl [1991] - Per la critica della filosofia del diritto di Hegel, in La questione ebraica, trad. di R. Panzieri, Roma, Editori Riuniti.

MARX, Karl [2003] - Le lotte di classe in Francia 1848-1856, www.marxists.org/italiano.

MARX, Karl [2004] - Manoscritti economico-filosofici del 1844, trad. e introd. di N. Bobbio, Torino, Einaudi.

MORONCINI, Bruno [1984] - Walter Benjamin e la moralità del moderno, Napoli, Guida.

MORONCINI, Bruno [2005] - Requiem per la scomparsa del padrone. il ruolo di Hegel nel dibattito sulla sovranità, in Arienzo - Caruso (a cura di), Conflitti, Napoli, Libreria Dante\&Descartes, 2005, 99.

PFERSMANN, Otto [2004] - Law's normativity in Spinoza's naturalism, in Cardozo law review, 25, 643-656.

REICH, Wilhelm [2002] - Psicologia di massa del fascismo, con un saggio di A. Zamperini, Torino, Einaudi.

REIK, Theodor [1953] - Le Masochisme, Paris, Payot.

RETIF DE LA BRETONNE, Nicolas [1784, 1972] - La paysanne pervertie, Paris, GarnierFlammarion.

RICEUR, Paul [1965] - De l’interprétation. Essai sur Freud, Paris, Seuil.

RICEEUR, Paul [2001] - Le juste 2, Paris, Esprit. 
RICEEUR, Paul [2006] - Le juste, la justice et son échec, Paris, Carnets l’Herne.

ROSENFELD, Michel [2000] - Interpretazioni. Il diritto fra etica e politica, Bologna, Il Mulino.

SAFATLE, Vladimir Pinheiro [2002] - L'acte au-delà de la Loi: Kant ave Sade comme point de torsion de la pensée lacanienne, in Essaim, n. 10, 2002, 73-106.

SOUSA SANTOS, Boaventura [2004] - Vers un Nouveau Sens Commun juridique. Droit, science et politique dans la transition paradigmatique, trad. Nathalie Gonzales Lajoie, Paris, LGDJ.

SPINOZA, Baruch [2005] - Etica. Trattato teologico-politico, a cura di Remo Cantoni e Franco Fergnani, Torino, UTET.

SUTTER, Laurent [2009] - Deleuze. La pratique du droit, Paris, Michalon.

TAYLOR, Charles [1998] - Il disagio della modernità, Bari-Roma, Laterza.

WELLMER, Albrecht [1986] - Ethik und Dialog. Elemente des moralischen Urteil bei Kant und in der Diskursethik, Krankfurt.

ŽIZEK, Slavoj [2001] - Kant and Sade: the ideal coup le, in http://lacan.com/frameXIII2.htm

ŽIZEK, Slavoj [1996] - The indivisble remainder, Londra, Verso.

ŽIZEK, Slavoj [2006] - Matrix, in La subjectivité à venir, Paris, Flammarion.

ŽIZEK, Slavoj [2007] - La sujet qui fâche. Le centre absent de l'ontologie politique, Paris, Flammarion.

Nata a Napoli, dove ha conseguito la laurea in giurisprudenza presso l'Università Federico II di Napoli, e dove ha svolto attività di docenza integrativa nel medesimo ateneo, ha poi conseguito il master di II livello in Mediazione e gestione alternativa dei conflitti presso l'istituto universitario di Napoli Suor Orsola Benincasa nonché il master 2 recherche in Droit public comparé européen, presso l’Université de Paris 1 Panthéon-Sorbonne. Ha tenuto convegni e seminari su diritti umani, teoria del diritto e diritto costituzionale in varie parti del mondo (Pekino, Shanghai, Berlino, Tolosa, Salisburgo, Brescia, Napoli), tra cui in Brasile, dove ha svolto anche attività di ricerca come pesquisadora estrangeira titolare della borsa di studi Capes-Fapergs dello Stato Rio grande do Sul presso l'università dell'Unisinos (2011). Svolge attività di ricerca all'Ecole des Hautes études en Sciences sociales di Parigi (Centre Georg Simmel), dove ha ottenuto il suo PhD in Droit et sciences sociales, e insegna diritto costituzionale presso l’Université de Paris Est-Créteil. Ha pubblicato, tra gli altri, Zéro. Révolution et critique de la Raison (2017); Imago of Revolution (2016); Peggio. La violenza, il Reale (2014); Un monde parfait. Géographies de l’Amérique imaginaire (2013); Monstres. Fantasmes, dieux, souverains (2012).E-mail: alessiamagliacane@gmail.com 